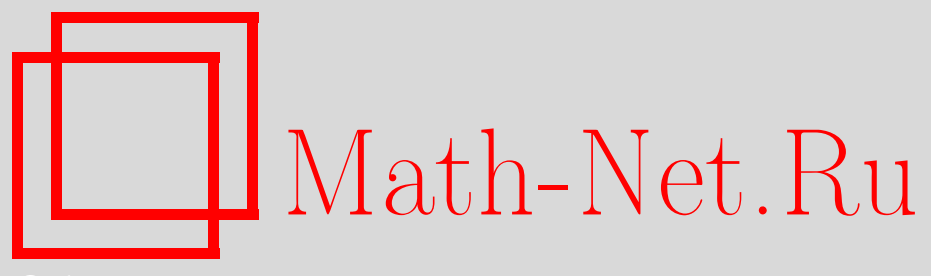

А. И. Двирный, В. И. Слынько, Об устойчивости по нелинейному квазиоднородному приближению дифференциальных уравнений с импульсным воздействием, Матем. сб., 2014, том 205, номер 6, 109-138

DOI: https://doi.org/10.4213/sm8233

Использование Общероссийского математического портала Math-Net.Ru подразумевает, что вы прочитали и согласны с пользовательским соглашением http://www . mathnet.ru/rus/agreement

Параметры загрузки:

IP : 3.81 .55 .215

26 апреля 2023 г., 18:12:29

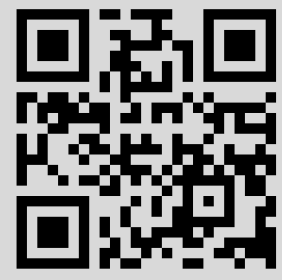




\author{
А. И. Двирный, В. И. Слынько
}

\title{
Об устойчивости по нелинейному квазиоднородному приближению дифференциальных уравнений с импульсным воздействием
}

\begin{abstract}
Для квазиоднородных систем дифференциальных уравнений с импульсным воздействием установлены обратные теоремы прямого метода Ляпунова. Получены условия существования функций Ляпунова, удовлетворяющих оценкам, характерным для квазиоднородных функций. C помощью этих результатов установлены условия устойчивости состояния равновесия нелинейной системы с импульсным воздействием на основе свойств ее квазиоднородного приближения. Полученные результаты проиллюстрированы на примере крупномасштабной системы с однородными подсистемами.
\end{abstract}

Библиография: 30 названий.

Ключевые слова: импульсное воздействие, квазиоднородная система, устойчивость по Ляпунову, прямой метод Ляпунова.

DOI: $10.4213 / \mathrm{sm} 8233$

\section{$\S 1$. Введение}

Исследование устойчивости критических положений равновесия нелинейных систем дифференциальных уравнений с импульсным воздействием (импульсных систем) является актуальной задачей для этого класса систем. Для систем обыкновенных дифференциальных уравнений при помощи принципа сведения (см. [1]-[6]) аналогичная задача может быть сведена к исследованию систем, которые содержат лишь критические переменные. Такие системы называют также модельными системами (см. [2]). Исследование модельных систем - существенно нелинейная задача, при решении которой важно в правой части системы выделить главную часть, которая и будет определять свойства устойчивости равновесия модельной системы. Эта главная часть может обладать свойствами однородности или квазиоднородности (см. [2], [7]). Например, к случаю квазиоднородной главной части приводит критический случай с кратным нулевым корнем, которому соответствует жорданова клетка порядка больше 1. Исследованию устойчивости по нелинейному приближению для систем обыкновенных дифференциальных уравнений посвящены работы И. Г. Малкина [8], Н. Н. Красовского [9], [10], В. И. Зубова [7], [11]. Для систем обыкновенных дифференциальных уравнений с квазиоднородной правой частью В. И. Зубов (см. [11]) установил условия устойчивости по нелинейному приближению, дополнительно предполагая, кроме условия асимптотической устойчивости состояния равновесия этой системы, еще и наличие некоторой априорной оценки 
решений. В основе этого результата лежит обратная теорема Ляпунова, утверждающая факт существования квазиоднородной функции Ляпунова с подходящими свойствами. При доказательстве этого результата существенно используется автомодельность автономной квазиоднородной системы относительно замены переменных $x \rightarrow \alpha^{G} x, t \rightarrow \alpha^{\mu-1} t$. Для систем обыкновенных дифференциальных уравнений с однородной правой частью Н. Н. Красовский [10] доказал, что из асимптотической устойчивости нулевого решения системы следует существование функции Ляпунова, удовлетворяющей оценкам, характерным для форм. В совокупности с результатами В. И. Зубова этот факт приводит к выводу о существовании однородной функции Ляпунова. Доказательство этих результатов также существенно опирается на свойство автомодельности однородной системы.

Для импульсных систем проблема устойчивости в критических случаях является менее разработанной. В работе [12] обоснован принцип сведения для импульсных систем в некоторых частных случаях. В работе [13] исследованы аналоги некоторых классических критических случаев, общий способ построения функции Ляпунова для импульсных систем в критических случаях представлен в [14]. Важным вопросом для теории устойчивости критических положений равновесия импульсных систем является исследование устойчивости по квазиоднородному приближению. Уместно отметить, что вопрос об устойчивости по линейному приближению для импульсных систем решен в монографии А. М. Самойленко и Н. А. Перестюка (см. [15]).

Основной отличительной особенностью квазиоднородных импульсных систем, в отличие от квазиоднородных систем обыкновенных дифференциальных уравнений, является отсутствие свойства автомодельности. Поэтому метод исследования устойчивости по квазиоднородному приближению, развитый В. И. Зубовым (см. [7], [11]), требует некоторой модификации. При этом можно использовать общие подходы к обращению теорем Ляпунова для импульсных систем, развитые в работе А. О. Игнатьева (см. [16]).

Настоящая работа состоит из шести параграфов. В 11 обоснована актуальность работы. Второй параграф посвящен формулировке постановки задачи и основного результата. В $\S 3$ доказана теорема о существовании функции Ляпунова для квазиоднородной импульсной системы. Четвертый параграф посвящен доказательству основной теоремы об устойчивости по квазиоднородному приближению. В $\S 5$ развиваются методы получения априорных оценок решений квазиоднородных и однородных импульсных систем. В $\S 6$ приведены примеры приложения полученных результатов к исследованию устойчивости критического положения равновесия крупномасштабной импульсной системы с однородными независимыми подсистемами. Показана общность полученных результатов.

\section{§ 2. Постановка задачи}

Пусть $\mathbb{R}^{n}-n$-мерное евклидово пространство с нормой $\|\cdot\|$. Обозначим $G=$ $\operatorname{diag}\left(g_{1}, \ldots, g_{n}\right)$, где $g_{s}=p_{s} / q_{s}, s=\overline{1, n}, p_{s}, q_{s}$ - натуральные числа, $q_{s}$ - нечетные числа. Для действительного числа $\alpha \in \mathbb{R}$ обозначим $\alpha^{G}=\operatorname{diag}\left(\alpha^{g_{1}}, \ldots, \alpha^{g_{n}}\right)$. 
Напомним (см. [7], [17]), что функция $f \in C\left(\mathbb{R}^{n} ; \mathbb{R}^{n}\right)$ называется квазиоднородной функцией измерения $\mu=p / q$ типа $G=\operatorname{diag}\left(g_{1}, \ldots, g_{n}\right)(p-$ натуральное число, $q$ - нечетное число), если при всех $x \in \mathbb{R}^{n}, \alpha \in \mathbb{R}$ выполняется равенство

$$
f\left(\alpha^{G} x\right)=\alpha^{G+(\mu-1) I} f(x) .
$$

Аналогично, функция $V \in C\left(\mathbb{R}^{n} ; \mathbb{R}\right)$ называется квазиоднородной измерения $m$ типа $G$, если при всех $\alpha \in \mathbb{R}, x \in \mathbb{R}^{n}$ справедливо равенство

$$
V\left(\alpha^{G} x\right)=\alpha^{m} V(x) .
$$

Следуя В. И. Зубову (см. [11]), введем также функцию $R(x)=\sum_{s=1}^{n}\left|x_{s}\right|^{1 / g_{s}}$. Отметим, что $R\left(\alpha^{G} x\right)=|\alpha| R(x)$ при всех $\alpha \in \mathbb{R}, x \in \mathbb{R}^{n}$. Множества $\mathscr{N}_{r}=$ $\left\{x \in \mathbb{R}^{n} \mid R(x)<r\right\}$ являются окрестностями точки $x=0$, хотя не всегда являются выпуклыми (поскольку $R(x)$ в общем случае нормой не является).

Для вектора $x=\left(x_{1}, \ldots, x_{n}\right)^{T}$ обозначим $|x|=\left(\left|x_{1}\right|, \ldots,\left|x_{n}\right|\right)^{T} \in \mathbb{R}_{+}^{n}$ и заметим, что из неравенств $y \geqslant x \geqslant 0$ следует неравенство $R(y) \geqslant R(x)$.

Также заметим, что при всех $\theta \in[0,1], \mathrm{x}_{i} \in \mathbb{R}^{n}, i=1,2$, справедливо неравенство

$$
R\left(\theta \mathrm{x}_{1}+(1-\theta) \mathrm{x}_{2}\right) \leqslant R\left(\left|\mathrm{x}_{1}\right|+\left|\mathrm{x}_{2}\right|\right) .
$$

Если $V \in C\left(\mathbb{R}^{n} ; \mathbb{R}\right)$ - квазиоднородная функция измерения $m$ типа $G$, то существуют действительные постоянные $\eta_{i}, i=1,2$, для которых выполняются неравенства (см. [11])

$$
\eta_{1} R^{m}(x) \leqslant V(x) \leqslant \eta_{2} R^{m}(x) .
$$

Если $\eta_{1}>0$, то функция $V(x)$ является положительно определенной.

Рассмотрим систему дифференциальных уравнений с импульсным воздействием

$$
\begin{gathered}
\frac{d x}{d t}=f(x)+P(t, x), \quad t \neq \tau_{k}, \\
\Delta x(t)=g(x(t))+Q_{k}(x(t)), \quad t=\tau_{k},
\end{gathered}
$$

где $x \in \mathbb{R}^{n}, f \in C^{1}\left(\mathbb{R}^{n} ; \mathbb{R}^{n}\right), g \in C\left(\mathbb{R}^{n} ; \mathbb{R}^{n}\right), \Delta x(t)=x(t+0)-x(t)$. Относительно функций $f$ и $g$ предположим, что они являются квазиоднородными функциями измерения $\mu$ типа $G$. Функции $P(t, x)$ и $Q_{k}(x)$ описывают некоторые возмущения правых частей квазиоднородного приближения системы

$$
\begin{gathered}
\frac{d y}{d t}=f(y), \quad t \neq \tau_{k}, \\
\Delta y(t)=g(y(t)), \quad t=\tau_{k} .
\end{gathered}
$$

Относительно функций $P(t, x)$ и $Q_{k}(x)$ предполагается, что они удовлетворяют условиям, гарантирующим существование и единственность решения задачи Коши $x\left(t ; t_{0}, x_{0}\right)$ для системы дифференциальных уравнений $(2.1)$. Кроме того, предположим, что решения $x\left(t ; t_{0}, x_{0}\right)$ непрерывны слева при $t=\tau_{k}$ и непрерывно зависят от начальных условий $x_{0}$. Также примем, что $P(t, 0)=0$, $Q_{k}(0)=0$.

Относительно моментов импульсного воздействия $\left\{\tau_{k}\right\}_{k=1}^{\infty}$ предположим, что они удовлетворяют двустороннему неравенству

$$
0<\theta_{1} \leqslant \tau_{k+1}-\tau_{k} \leqslant \theta_{2}<+\infty .
$$


Целью настоящей работы является установление условий, при которых из асимптотической устойчивости состояния равновесия $y=0$ системы квазиоднородного приближения (2.2) следует асимптотическая устойчивость состояния равновесия $x=0$ возмущенной системы (2.1). Аналогичная задача рассматривалась также в работах [16], [18], [19]. В этих работах получены условия обращения теорем прямого метода Ляпунова для импульсных систем при достаточно общих предположениях относительно правых частей системы. В качестве приложения полученных результатов указаны условия, при которых устойчивость тривиального решения системы сохраняется при малых возмущениях правых частей. Эти условия состоят в том, что возмущения являются асимптотически затухающими (или асимптотически затухающими "в среднем") при возрастании времени. В настоящей работе рассматривается более узкий класс импульсных систем - системы с квазиоднородными правыми частями. За счет такого сужения класса систем удается доказать существование функции Ляпунова со свойствами, аналогичными свойствам квазиоднородных функций. С помощью таких функций можно установить условия сохранения асимптотической устойчивости тривиального состояния равновесия в случае, когда возмущения являются достаточно малыми (равномерно по времени) в некоторой окрестности состояния равновесия. Эти условия не являются следствием результатов работ [16], [18], [19]. Некоторые из результатов, полученные в настоящей работе, являются развитием результатов работ [16], [18], [19].

Рассмотрим однопараметрическое семейство дифференциальных уравнений с импульсным воздействием

$$
\frac{d z}{d \tau}=f(z), \quad \tau \neq \varepsilon^{\mu-1} \tau_{k}, \quad \Delta z(\tau)=\varepsilon^{\mu-1} g(z(\tau)), \quad \tau=\varepsilon^{\mu-1} \tau_{k},
$$

где $z \in \mathbb{R}^{n}, \varepsilon \in(0,1], z\left(\tau ; \tau_{0}, y_{0}, \varepsilon\right)$ - решение задачи Коши для системы $(2.3)$, $z\left(\tau_{0} ; \tau_{0}, z_{0}, \varepsilon\right)=z_{0}$.

Основной результат настоящей работы состоит в следующем утверждении.

Предположим, что для решений системы (2.3) выполняется следующая априорная оценка: существуют положительные постоянные $a, \sigma, \tau^{\prime}, r_{0}$, не зависящие от $\varepsilon \in(0,1]$ и $\tau_{0}$ и такие, что при всех $\tau \geqslant \tau_{0}+\tau^{\prime}$ и $z_{0} \in \partial \mathscr{N}_{r_{0}}$, где $\partial \mathscr{N}_{r_{0}}$ граница множества $\mathscr{N}_{r_{0}}$, выполняется неравенство

$$
\left\|z\left(\tau ; \tau_{0}, z_{0}, \varepsilon\right)\right\| \leqslant a\left(\tau-\tau_{0}\right)^{-\sigma} .
$$

ЗАмЕчАниЕ. Нетрудно видеть, что выполнение априорной оценки (2.4) для решений системы $(2.2)$ при $\varepsilon=1$ с показателем $\sigma=1 /(\mu-1)$ влечет за собой выполнение априорной оценки (2.4) с тем же показателем при любом $\varepsilon \in(0,1]$.

Теорема 1. Пусть система квазиоднородного приближения (2.2) такова, что ее состояние равновесия $y=0$ является асимптотически устойчивым, для однопараметрического семейства квазиоднородных систем (2.3) выполняется априорная оценка (2.4), а для возмущений $P(t, x)$ и $Q_{k}(x)$ равномерно nо $t, k$ выполняются предельные соотношения

$$
\frac{\|P(t, x)\|}{\left\|R^{(\mu-1) I+G}(x)\right\|} \rightarrow 0, \quad \frac{\left\|Q_{k}(x)\right\|}{\left\|R^{(\mu-1) I+G}(x)\right\|} \rightarrow 0
$$

$n p u\|x\| \rightarrow 0$. 
Тогда состояние равновесия $x=0$ системы (2.1) асимптотически устойчиво.

Доказательству этой теоремы посвящен $\S 4$.

\section{§ 3. Вспомогательные результаты}

Исследуем вопрос о существовании вспомогательной функции Ляпунова для системы нелинейного приближения (2.2) в случае, когда состояние равновесия $y=0$ является асимптотически устойчивым. При этом используем некоторые идеи работ [7], [16].

Напомним некоторые общие сведения, касающиеся теорем метода сравнения для систем дифференциальных уравнений с импульсным воздействием, которые будут использоваться в дальнейшем изложении. Множество

$$
\mathbb{R}_{+}^{n}=\left\{x=\left(x_{1}, \ldots, x_{n}\right)^{T} \in \mathbb{R}^{n} \mid x_{i} \geqslant 0, i=\overline{1, n}\right\}
$$

является телесным конусом в пространстве $\mathbb{R}^{n}$. Этот конус естественным образом вводит в пространстве $\mathbb{R}^{n}$ полуупорядоченность по правилу: $x \geqslant y$, если и только если $x-y \in \mathbb{R}_{+}^{n}$. Пусть $\Omega$ - связная окрестность точки $x=0$, $\Omega_{1}=\Omega \cap \mathbb{R}_{+}^{n}$.

ОПРЕДЕЛЕНИЕ 1. Функция $\varphi: \Omega_{1} \rightarrow \mathbb{R}^{n}$ называется неубъвающей, если из условия $u_{2} \geqslant u_{1}, u_{i} \in \Omega_{1}$, следует, что $\varphi\left(u_{2}\right) \geqslant \varphi\left(u_{1}\right)$.

ОПРЕДЕЛЕНИЕ 2 (см. [20]). Функция $\varphi: \mathbb{R} \times \Omega_{1} \rightarrow \mathbb{R}^{n}$ удовлетворяет условиям Важевского (называется квазимонотонно неубывающей), если каждая из компонент $\varphi_{s}(t, x), s=\overline{1, n}$, вектора $\varphi(t, x)$ удовлетворяет условию: из

$$
0 \leqslant x_{k}^{\prime} \leqslant x_{k}^{\prime \prime}, \quad k \neq i, \quad k=\overline{1, n}, \quad x_{i}^{\prime}=x_{i}^{\prime \prime}
$$

следует, что $\varphi_{i}\left(t, x^{\prime}\right) \leqslant \varphi_{i}\left(t, x^{\prime \prime}\right)$.

Рассмотрим систему дифференциальных уравнений с импульсным воздействием

$$
\begin{gathered}
\frac{d u}{d t}=G(t, u), \quad t \neq \tau_{k}, \\
\Delta u(t)=\Psi_{k}(u), \quad t=\tau_{k},
\end{gathered}
$$

где $u \in \mathbb{R}^{n}, G \in C\left(\mathbb{R} \times \Omega_{1} ; \mathbb{R}^{n}\right), \Psi_{k} \in C\left(\Omega_{1} ; \mathbb{R}^{n}\right)$. Предположим, что решение задачи Коши для системы уравнений (3.1) единственно. Пусть вектор-функция $u:\left[t_{0}, \omega\right) \rightarrow \mathbb{R}^{n}$ является решением системы (3.1) с начальным условием $u\left(t_{0}\right)=u_{0},\left(t_{0}, u_{0}\right) \in \mathbb{R} \times \Omega_{1}$ и $u\left(\tau_{k}+0\right) \in \Omega_{1}$, если только $\tau_{k} \in\left(t_{0}, \omega\right)$. Пусть $m:\left[t_{0}, \widetilde{\omega}\right) \rightarrow \mathbb{R}^{n}, \widetilde{\omega} \leqslant \omega,-$ кусочно непрерывная вектор-функция с разрывами первого рода в точках $\tau_{k}$, непрерывная слева от них и удовлетворяющая условиям

а) $m\left(\tau_{k}+0\right) \in \Omega_{1}$, если $\tau_{k} \in\left[t_{0}, \widetilde{\omega}\right)$;

б) $D^{+} m(t) \leqslant G(t, m(t)), t \neq \tau_{k}, \Delta m(t) \leqslant \Psi_{k}(m(t)), t=\tau_{k}, k \in \mathbb{N}, m\left(t_{0}\right) \leqslant u_{0}$. Здесь $D^{+}-$верхняя производная Дини.

ЛЕмма 1 (см. [21]). Предположим, что

1) функиия $G$ - квазимонотонно неубъвающая на множестве $\mathbb{R} \times \Omega_{1}$; 
2) функиии $u+\Psi_{k}(u), k \in \mathbb{N},-$ неубъвающие на множестве $\Omega_{1}$;

3) выполняются условия а) $u$ б).

Тогда $m(t) \leqslant u(t)$ при всех $t \in\left[t_{0}, \widetilde{\omega}\right)$.

При выполнении условий 1$)$ и 2$)$ леммы 1 оператор сдвига $U\left(t, t_{0}\right): \Omega_{1} \rightarrow \mathbb{R}^{n}$ вдоль решений системы (3.1) является неубывающим, т.е. из условий $u_{2} \geqslant$ $u_{1} \geqslant 0$ следует, что $U\left(t, t_{0}\right) u_{2} \geqslant U\left(t, t_{0}\right) u_{1}$ при всех $t \geqslant t_{0}$, для которых определены правая и левая части этого неравенства и $U\left(\tau_{k}, t_{0}\right) u_{i} \in \Omega_{1}, i=1,2$. Этот факт является следствием аналогичного утверждения для систем обыкновенных дифференциальных уравнений, правые части которых удовлетворяют условию Важевского (см. [22]).

Сформулируем еще один результат, необходимый для дальнейшего изложения, заимствованный из работы [16].

Лемма 2. Пусть $t_{0} \in \mathbb{R}, f_{1} \in\left[t_{0},+\infty\right) \rightarrow \mathbb{R}_{+}, f_{2} \in\left[t_{0},+\infty\right) \rightarrow \infty-$ две ограниченные неотрицательные кусочно непрерывные функиии, имеющие односторонние пределы в точках разрыва, такие, что

$$
\lim _{t \rightarrow+\infty} f_{1}(t)=\lim _{t \rightarrow+\infty} f_{2}(t)=0 .
$$

Тогда справедливо неравенство

$$
\left|\sup _{t \in\left[t_{0},+\infty\right)} f_{1}(t)-\sup _{t \in\left[t_{0},+\infty\right)} f_{2}(t)\right| \leqslant \sup _{t \in\left[t_{0},+\infty\right)}\left|f_{1}(t)-f_{2}(t)\right| .
$$

Отметим справедливость тождества

$$
y\left(t ; t_{0}, \varepsilon^{G} y_{0}\right)=\varepsilon^{G} z\left(\varepsilon^{\mu-1} t ; \varepsilon^{\mu-1} t_{0}, y_{0}, \varepsilon\right)
$$

при всех $t \geqslant t_{0}$, для которых правая и левая части равенства существуют. Здесь $y\left(t ; t_{0}, y_{0}\right)$ - решение задачи Коши для системы $(2.2)$.

Действительно, пусть

$$
\zeta(t)=y\left(t ; t_{0}, \varepsilon^{G} y_{0}\right)-\varepsilon^{G} z\left(\varepsilon^{\mu-1} t ; \varepsilon^{\mu-1} t_{0}, y_{0}, \varepsilon\right),
$$

тогда функция $\zeta(t)$ является решением системы дифференциальных уравнений с импульсным воздействием

$$
\begin{gathered}
\frac{d \zeta}{d t}=f\left(\zeta+\varepsilon^{G} z\left(\varepsilon^{\mu-1} t ; \varepsilon^{\mu-1} t_{0}, y_{0}, \varepsilon\right)\right)-\varepsilon^{G+(\mu-1) I} f\left(z\left(\varepsilon^{\mu-1} t ; \varepsilon^{\mu-1} t_{0}, y_{0}, \varepsilon\right)\right), \\
t \neq \tau_{k}, \\
\Delta \zeta(t)=g\left(\zeta+\varepsilon^{G} z\left(\varepsilon^{\mu-1} t ; \varepsilon^{\mu-1} t_{0}, y_{0}, \varepsilon\right)\right)-\varepsilon^{G+(\mu-1) I} g\left(z\left(\varepsilon^{\mu-1} t ; \varepsilon^{\mu-1} t_{0}, y_{0}, \varepsilon\right)\right), \\
t=\tau_{k} .
\end{gathered}
$$

В силу предположения о квазиоднородности функций $f$ и $g$ система уравнений (3.3) имеет решение $\zeta=0$. Поскольку $\zeta\left(t_{0}\right)=0$, то в силу единственности решений задачи Коши $\zeta(t) \equiv 0$ при всех $t \geqslant t_{0}$, для которых правая и левая части равенства (3.2) существуют.

ТЕОРема 2. Предположим, что состояние равновесия у = 0 системы (2.2) асимптотически устойчиво и для однопараметрического семейства систем (2.3) выполняется априорная оченка (2.4). 
Тогда для достаточно большого рачионального числа $m\left(m=s_{1} / s, s-\right.$ нечетное число, $s_{1}$ - четное число) и положительно определенной квазиоднородной функиии $W(y)$ m-го измерения типа $G$ существуют функция $V(t, y)$, положительные постоянные $r_{1}, \gamma_{i}, i=1,2,3$, такие, что выполняются оченки

$$
\begin{gathered}
\gamma_{1} R^{m+1-\mu}(y) \leqslant V(t, y) \leqslant \gamma_{2} R^{m+1-\mu}(y), \\
\left.D^{+} V(t, y)\right|_{(2.2)} \leqslant-W(y), \quad t \neq \tau_{k}, \\
V\left(\tau_{k}+0, y+g(y)\right) \leqslant V\left(\tau_{k}, y\right), \quad k \in \mathbb{N}, \\
\left|V\left(t, y_{2}\right)-V\left(t, y_{1}\right)\right| \leqslant \gamma_{3}\left\|R^{(m-\mu+1) I-G}\left(\left|y_{1}\right|+\left|y_{2}\right|\right)\right\|\left\|y_{2}-y_{1}\right\|
\end{gathered}
$$

при всех $y, y_{1}, y_{2} \in \mathscr{N}_{r_{1}}$.

ДокАЗАТЕЛЬСтво. Доказательство этой теоремы проведем по следующей схеме: сначала построим вспомогательную функцию $V(t, x)$, удовлетворяющую оценкам (3.4) и (3.5). Затем, используя эту вспомогательную функцию, уточним оценки для решений импульсной системы (2.2). И, наконец, докажем оценку (3.6).

При доказательстве этой теоремы удобно считать, что $g_{1} \leqslant g_{2} \leqslant \cdots \leqslant g_{n}$. Прежде всего напомним, что существуют положительные числа $\eta_{i}, i=1,2$, такие, что при всех $y \in \mathbb{R}^{n}$ выполняются неравенства

$$
\eta_{1} R^{m}(y) \leqslant W(y) \leqslant \eta_{2} R^{m}(y) .
$$

Следуя идеям работ [11], [16], рассмотрим функцию

$$
V\left(t_{0}, y_{0}\right)=\int_{t_{0}}^{+\infty} W\left(y\left(s ; t_{0}, y_{0}\right)\right) d s+\sup _{s \in\left[t_{0},+\infty\right)} R^{m+1-\mu}\left(y\left(s ; t_{0}, y_{0}\right)\right) .
$$

Докажем сходимость несобственного интеграла, входящего в правую часть равенства (3.8). Будем считать, что $y_{0} \in \mathscr{N}_{r_{1}}$, и обозначим $\varepsilon_{y_{0}}=R\left(y_{0}\right) / r_{0} \in(0,1]$, $\tau_{0}=\varepsilon_{y_{0}}^{\mu-1} t_{0}$. Тогда из тождества (3.2) следует, что

$$
y\left(s ; t_{0}, y_{0}\right)=\varepsilon_{y_{0}}^{G} z\left(\varepsilon_{y_{0}}^{\mu-1} s ; \varepsilon_{y_{0}}^{\mu-1} t_{0}, \varepsilon_{y_{0}}^{-G} y_{0}, \varepsilon_{y_{0}}\right) .
$$

Поэтому

$$
\begin{gathered}
\int_{t_{0}}^{+\infty} W\left(y\left(s ; t_{0}, y_{0}\right)\right) d s=\varepsilon_{y_{0}}^{m+1-\mu} \int_{\tau_{0}}^{+\infty} W\left(z\left(\tau ; \tau_{0}, \varepsilon_{y_{0}}^{-G} y_{0}, \varepsilon_{y_{0}}\right)\right) d \tau \\
=\varepsilon_{y_{0}}^{m+1-\mu}\left(\int_{\tau_{0}}^{\tau_{0}+\tau^{\prime}} W\left(z\left(\tau ; \tau_{0}, \varepsilon_{y_{0}}^{-G} y_{0}, \varepsilon_{y_{0}}\right)\right) d \tau\right. \\
\left.\quad+\int_{\tau_{0}+\tau^{\prime}}^{+\infty} W\left(z\left(\tau ; \tau_{0}, \varepsilon_{y_{0}}^{-G} y_{0}, \varepsilon_{y_{0}}\right)\right) d \tau\right) .
\end{gathered}
$$

Используя обобщенную лемму Гронуолла-Беллмана (см. [15]), можно показать, что существует положительная постоянная $M_{\tau^{\prime}}$, не зависящая от $\varepsilon \in(0,1]$ и зависящая от $\tau^{\prime}$, такая, что при всех $s \in\left[\tau_{0}, \tau_{0}+\tau^{\prime}\right]$ выполняется неравенство

$$
\left\|z\left(s ; \tau_{0}, z_{0}, \varepsilon\right)\right\| \leqslant M_{\tau^{\prime}}\left\|z_{0}\right\| .
$$


Поэтому при всех $s \in\left[\tau_{0}, \tau_{0}+\tau^{\prime}\right]$ выполняется неравенство

$$
\begin{aligned}
& W\left(z\left(s ; \tau_{0}, \varepsilon_{y_{0}}^{-G} y_{0}, \varepsilon_{y_{0}}\right)\right) \leqslant \eta_{2} R^{m}\left(z\left(s ; \tau_{0}, \varepsilon_{y_{0}}^{-G} y_{0}, \varepsilon_{y_{0}}\right)\right) \\
& \quad \leqslant \eta_{2}\left(\sum_{s=1}^{n} M_{\tau^{\prime}}^{\frac{1}{g_{s}}}\left\|\varepsilon_{y_{0}}^{-G} y_{0}\right\|^{\frac{1}{g_{s}}}\right)^{m} \leqslant \eta_{2} M^{*}\left(\sum_{l=1}^{n}\left(\sum_{s=1}^{n} r_{0}^{2 g_{s}}\right)^{\frac{g_{l}}{2}}\right)^{m} .
\end{aligned}
$$

Здесь обозначено $M^{*}=\max _{s=\overline{1, n}} M_{\tau^{\prime}}^{\frac{m}{g_{s}}}$ и учтено $R\left(\varepsilon_{y_{0}}^{-G} y_{0}\right)=\varepsilon_{y_{0}}^{-1} R\left(y_{0}\right)=r_{0}$.

Как следствие, получим неравенство

$$
\int_{\tau_{0}}^{\tau_{0}+\tau^{\prime}} W\left(z\left(\tau ; \tau_{0}, \varepsilon_{y_{0}}^{-G} y_{0}, \varepsilon_{y_{0}}\right)\right) d \tau \leqslant \tau^{\prime} \eta_{2} M^{*}\left(\sum_{l=1}^{n}\left(\sum_{s=1}^{n} r_{0}^{2 g_{s}}\right)^{\frac{g_{l}}{2}}\right)^{m}
$$

Аналогично, с учетом априорной оценки (2.4) получим

$$
\begin{gathered}
\int_{\tau_{0}+\tau^{\prime}}^{+\infty} W\left(z\left(\tau ; \tau_{0}, \varepsilon_{y_{0}}^{-G} y_{0}, \varepsilon_{y_{0}}\right)\right) d \tau \leqslant \eta_{2} \int_{\tau_{0}+\tau^{\prime}}^{+\infty} R^{m}\left(z\left(\tau ; \tau_{0}, \varepsilon_{y_{0}}^{-G} y_{0}, \varepsilon_{y_{0}}\right)\right) d \tau \\
\leqslant \eta_{2} \int_{\tau_{0}+\tau^{\prime}}^{+\infty}\left(\sum_{s=1}^{n} a^{\frac{1}{g_{s}}}\left(\tau-\tau_{0}\right)^{-\frac{\sigma}{g_{s}}}\right)^{m} d \tau \\
\leqslant \eta_{2}\left(\sum_{s=1}^{n} a^{\frac{1}{g_{s}}-\frac{1}{g_{n}}}\left(\tau^{\prime}\right)^{\frac{\sigma}{g_{n}}-\frac{\sigma}{g_{s}}}\right)^{m} \int_{\tau_{0}+\tau^{\prime}}^{+\infty}\left(\tau-\tau_{0}\right)^{-\frac{m \sigma}{g_{n}}} d \tau .
\end{gathered}
$$

Выберем число $m$ так, чтобы выполнялось неравенство $m \sigma>g_{n}$, тогда

$$
\int_{\tau_{0}+\tau^{\prime}}^{+\infty}\left(\tau-\tau_{0}\right)^{-\frac{m \sigma}{g_{n}}} d \tau=\frac{\left(\tau^{\prime}\right)^{1-\frac{m \sigma}{g_{n}}}}{m \sigma / g_{n}-1} .
$$

Этим доказана сходимость несобственного интеграла, входящего в выражение для функции $V\left(t_{0}, y_{0}\right)$. Покажем конечность величины

$$
\sup _{s \in\left[t_{0},+\infty\right)} R^{m+1-\mu}\left(y\left(s ; t_{0}, y_{0}\right)\right)
$$

Действительно,

$$
\sup _{s \in\left[t_{0},+\infty\right)} R^{m+1-\mu}\left(y\left(s ; t_{0}, y_{0}\right)\right)=\varepsilon_{y_{0}}^{m+1-\mu} \sup _{\tau \in\left[t_{0},+\infty\right)} R^{m+1-\mu}\left(z\left(\tau ; \tau_{0}, \varepsilon_{y_{0}}^{-G} y_{0}, \varepsilon_{y_{0}}\right)\right) .
$$

Из априорной оценки (2.4) и неравенства (3.9) следует оценка

$$
R\left(z\left(\tau ; \tau_{0}, \varepsilon_{y_{0}}^{-G} y_{0}, \varepsilon_{y_{0}}\right)\right) \leqslant \begin{cases}M^{*} \sum_{l=1}^{n}\left(\sum_{s=1}^{n} r_{0}^{2 g_{s}}\right)^{\frac{g_{l}}{2}}, & \tau \in\left[\tau_{0}, \tau_{0}+\tau^{\prime}\right], \\ \sum_{s=1}^{n}\left(a M_{\tau^{\prime}}\right)^{\frac{1}{g_{s}}}\left(\tau^{\prime}\right)^{-\frac{\sigma}{g_{s}}}, & \tau \in\left(\tau_{0}+\tau^{\prime},+\infty\right) .\end{cases}
$$

Таким образом, функция $V(t, y)$ определена при всех $(t, y) \in \mathbb{R} \times \overline{\mathscr{N}}_{r_{1}}$. Из приведенных выше рассуждений следуют неравенства

$$
V(t, y) \leqslant \gamma_{2} R^{m+1-\mu}(y), \quad V(t, y) \geqslant R^{m+1-\mu}(y) .
$$


Существование верхней производной Дини $\left.D^{+} V(t, y)\right|_{(2.2)}$ вдоль решений системы дифференциальных уравнений $(2.2)$ при $t \neq \tau_{k}$ и выполнение неравенств (3.5) фактически доказано в работе [16].

Полученные оценки (3.4)-(3.5) для функции $V(t, y)$ и лемма 1 позволяют несколько уточнить оценки для решений системы $(2.2)$. Зафиксируем число $m_{0}$ настолько большим, чтобы оценки (3.4)-(3.5) для функции $V(t, y)$ выполнялись при $m=m_{0}$. Лемма 1 позволяет установить оценку

$$
V\left(t, y\left(t ; t_{0}, y_{0}\right)\right) \leqslant U\left(t ; t_{0}, V\left(t_{0}, y_{0}\right)\right),
$$

где $U\left(t ; t_{0}, U_{0}\right)$ - решение задачи Коши для дифференциального уравнения с импульсным воздействием

$$
\begin{gathered}
\frac{d U}{d t}=-\frac{\eta_{1}}{\gamma_{2}^{\frac{m_{0}}{m_{0}+1-\mu}}} U^{\frac{m_{0}}{m_{0}+1-\mu}}(t), \quad t \neq \tau_{k}, \\
\Delta U(t)=0, \quad t=\tau_{k},
\end{gathered}
$$

которое является обыкновенным дифференциальным уравнением. Обозначим

$$
\eta_{3}=\frac{\eta_{1}}{\gamma_{2}^{\frac{m_{0}}{m_{0}+1-\mu}}}
$$

тогда из неравенств (3.10) следует

$$
\begin{aligned}
R\left(y\left(t ; t_{0}, y_{0}\right)\right) & \leqslant U^{\frac{1}{m_{0}+1-\mu}}\left(t ; t_{0}, \gamma_{2} R^{m_{0}+1-\mu}\left(y_{0}\right)\right) \\
& \leqslant R\left(y_{0}\right)\left(\gamma_{2}^{1-\mu}+\frac{\eta_{3}(\mu-1)}{m_{0}+1-\mu} R^{\mu-1}\left(y_{0}\right)\left(t-t_{0}\right)\right)^{\frac{1}{1-\mu}} .
\end{aligned}
$$

Обозначим

$$
\eta_{3}^{\prime}=\frac{\eta_{3}(\mu-1)}{m_{0}+1-\mu}
$$

тогда последнее неравенство можно представить в виде

$$
R\left(y\left(t ; t_{0}, y_{0}\right)\right) \leqslant \gamma_{2} R\left(y_{0}\right)\left(1+\eta_{3}^{\prime}\left(\gamma_{2} R\left(y_{0}\right)\right)^{\mu-1}\left(t-t_{0}\right)\right)^{\frac{1}{1-\mu}} .
$$

Докажем, что число $m$ можно увеличить так, чтобы выполнялось неравенство (3.6). При этом, не умаляя общности, будем считать, что $t_{0}<\tau_{1}$.

Действительно, с учетом того, что $y\left(s ; t_{0}, y_{i}\right) \rightarrow 0, i=1,2$, при $s \rightarrow+\infty$ из утверждения леммы 2 получим оценку

$$
\begin{aligned}
\mid V\left(t_{0}, y_{2}\right) & -V\left(t_{0}, y_{1}\right)\left|\leqslant \int_{t_{0}}^{+\infty}\right| W\left(y\left(s ; t_{0}, y_{2}\right)\right)-W\left(y\left(s ; t_{0}, y_{1}\right)\right) \mid d s \\
& +\sup _{s \in\left[t_{0},+\infty\right)}\left|R^{m+1-\mu}\left(y\left(s ; t_{0}, y_{2}\right)\right)-R^{m+1-\mu}\left(y\left(s ; t_{0}, y_{1}\right)\right)\right|
\end{aligned}
$$

Из формулы конечных приращений получим

$W\left(y\left(s ; t_{0}, y_{2}\right)\right)-W\left(y\left(s ; t_{0}, y_{1}\right)\right)=\int_{0}^{1}\left(\frac{\partial W}{\partial y}\left(y\left(s ; t_{0}, y_{\theta}\right)\right)^{T} \frac{\partial y}{\partial y_{0}}\left(s ; t_{0}, y_{\theta}\right) d \theta\left(y_{2}-y_{1}\right)\right.$,

где $y_{\theta}=y_{1}+\theta\left(y_{2}-y_{1}\right), \theta \in[0,1]$. 
Прежде всего отметим, что матрица Якоби

$$
J\left(t, t_{0}, y_{\theta}\right)=\frac{\partial y}{\partial y_{0}}\left(t ; t_{0}, y_{\theta}\right), \quad J\left(t_{0}, t_{0}, y_{\theta}\right)=I
$$

при $t \geqslant t_{0}$ удовлетворяет линейной системе дифференциальных уравнений с импульсным воздействием (системе уравнений в вариациях [15])

$$
\begin{aligned}
\frac{d J}{d t} & =\frac{\partial f}{\partial y}\left(y\left(t ; t_{0}, y_{\theta}\right)\right) J, & t & \neq \tau_{k}, \\
\Delta J & =\frac{\partial g}{\partial y}\left(y\left(t ; t_{0}, y_{\theta}\right)\right) J, & t & =\tau_{k} .
\end{aligned}
$$

Пусть $\xi \in \mathbb{R}^{n},\|\xi\|=1$ и

$$
\omega(t)=\left\|J\left(t, t_{0}, y_{\theta}\right) \xi\right\|^{2}, \quad \eta_{4}=\sup _{x \in \partial \mathscr{N}_{1}}\left\|\frac{\partial f}{\partial x}\right\|, \quad \eta_{5}=\sup _{x \in \partial \mathscr{N}_{1}}\left\|\frac{\partial g}{\partial x}\right\|,
$$

тогда вследствие свойства квазиоднородности функций $f$ и $g$ выполняются неравенства

$$
\left\|\frac{\partial f}{\partial y}(y)\right\| \leqslant \eta_{4} R^{\mu-1}(y), \quad\left\|\frac{\partial g}{\partial y}(y)\right\| \leqslant \eta_{5} R^{\mu-1}(y)
$$

и из (3.13) следуют неравенства

$$
\begin{aligned}
\frac{d \omega}{d t} & =\xi^{T} J^{T}\left(\frac{\partial f}{\partial y}\left(y\left(t ; t_{0}, y_{\theta}\right)\right)+\frac{\partial f^{T}}{\partial y}\left(y\left(t ; t_{0}, y_{\theta}\right)\right)\right) J \xi \leqslant 2\left\|\frac{\partial f}{\partial y}\left(y\left(t ; t_{0}, y_{\theta}\right)\right)\right\|\|J \xi\|^{2} \\
& \leqslant 2 \eta_{4} R^{\mu-1}\left(y\left(t ; t_{0}, y_{\theta}\right)\right) \omega(t), \quad t \neq \tau_{k}, \\
\omega\left(\tau_{k}+0\right) & =\xi^{T} J^{T}\left(I+\frac{\partial g}{\partial y}\left(y\left(\tau_{k} ; t_{0}, y_{\theta}\right)\right)\right)^{T}\left(I+\frac{\partial g}{\partial y}\left(y\left(\tau_{k} ; t_{0}, y_{\theta}\right)\right)\right) J \xi \\
& \leqslant\left(1+\left\|\frac{\partial g}{\partial y}\left(y\left(\tau_{k} ; t_{0}, y_{\theta}\right)\right)\right\|\right)^{2} \omega\left(\tau_{k}\right) \leqslant\left(1+\eta_{5} R^{\mu-1}\left(y\left(\tau_{k} ; t_{0}, y_{\theta}\right)\right)\right)^{2} \omega\left(\tau_{k}\right) .
\end{aligned}
$$

Применяя утверждение леммы 1 к функции $\omega(t)$, получим неравенство

$$
\begin{aligned}
\left\|J\left(t, t_{0}, y_{\theta}\right)\right\| & \leqslant \exp \left\{\eta_{4} \int_{t_{0}}^{t} R^{\mu-1}\left(y\left(s ; t_{0}, y_{\theta}\right)\right) d s\right\} \prod_{k=1}^{N\left(t_{0}, t\right)}\left(1+\eta_{5} R^{\mu-1}\left(y\left(\tau_{k} ; t_{0}, y_{\theta}\right)\right)\right) \\
& \leqslant \exp \left\{\eta_{4} \int_{t_{0}}^{t} R^{\mu-1}\left(y\left(s ; t_{0}, y_{\theta}\right)\right) d s+\eta_{5} \sum_{k=1}^{N\left(t_{0}, t\right)} R^{\mu-1}\left(y\left(\tau_{k} ; t_{0}, y_{\theta}\right)\right)\right\}
\end{aligned}
$$

где $N\left(t_{0}, t\right)$ - мощность множества $\left\{\tau_{k}\right\}_{k=1}^{\infty} \cap\left(t_{0}, t\right)$.

Применяя неравенство (3.11), получим оценки

$$
\begin{aligned}
\int_{t_{0}}^{t} R^{\mu-1}\left(y\left(s ; t_{0}, y_{\theta}\right)\right) d s \leqslant\left(\gamma_{2} R\left(y_{\theta}\right)\right)^{\mu-1} \int_{t_{0}}^{t} \frac{d s}{1+\eta_{3}^{\prime}\left(\gamma_{2} R\left(y_{\theta}\right)\right)^{\mu-1}\left(s-t_{0}\right)} \\
=\frac{1}{\eta_{3}^{\prime}} \ln \left(1+\eta_{3}^{\prime}\left(\gamma_{2} R\left(y_{\theta}\right)\right)^{\mu-1}\left(t-t_{0}\right)\right),
\end{aligned}
$$




$$
\begin{aligned}
& \sum_{k=1}^{N\left(t_{0}, t\right)} R^{\mu-1}\left(y\left(\tau_{k} ; t_{0}, y_{\theta}\right)\right) \leqslant\left(\gamma_{2} R\left(y_{\theta}\right)\right)^{\mu-1} \sum_{k=1}^{N\left(t_{0}, t\right)} \frac{1}{1+\eta_{3}^{\prime}\left(\gamma_{2} R\left(y_{\theta}\right)\right)^{\mu-1}\left(\tau_{k}-t_{0}\right)} \\
& \leqslant\left(\gamma_{2} R\left(y_{\theta}\right)\right)^{\mu-1}\left(1+\sum_{k=2}^{N\left(t_{0}, t\right)} \frac{1}{\tau_{k}-\tau_{k-1}} \int_{\tau_{k-1}}^{\tau_{k}} \frac{d s}{1+\eta_{3}^{\prime}\left(\gamma_{2} R\left(y_{\theta}\right)\right)^{\mu-1}\left(s-t_{0}\right)}\right) \\
& \quad \leqslant\left(\gamma_{2} R\left(y_{\theta}\right)\right)^{\mu-1}\left(1+\sum_{k=2}^{N\left(t_{0}, t\right)} \frac{1}{\theta_{1}} \int_{\tau_{k-1}}^{\tau_{k}} \frac{d s}{1+\eta_{3}^{\prime}\left(\gamma_{2} R\left(y_{\theta}\right)\right)^{\mu-1}\left(s-t_{0}\right)}\right) \\
& \leqslant\left(\gamma_{2} R\left(y_{\theta}\right)\right)^{\mu-1}+\frac{1}{\theta_{1} \eta_{3}^{\prime}} \ln \left(1+\eta_{3}^{\prime}\left(\gamma_{2} R\left(y_{\theta}\right)\right)^{\mu-1}\left(\tau_{N\left(t_{0}, t\right)}-t_{0}\right)\right) \\
& \leqslant\left(\gamma_{2} R\left(\left|y_{1}\right|+\left|y_{2}\right|\right)\right)^{\mu-1}+\frac{1}{\theta_{1} \eta_{3}^{\prime}} \ln \left(1+\eta_{3}^{\prime}\left(\gamma_{2} R\left(y_{\theta}\right)\right)^{\mu-1}\left(t-t_{0}\right)\right)
\end{aligned}
$$

Последнее неравенство справедливо при всех $t \geqslant t_{0}$, если дополнительно принять, что при $N\left(t_{0}, t\right)=0$ сумма в пределах от 1 до $N\left(t_{0}, t\right)$ равна нулю.

Обозначим $M=\gamma_{2} \sup _{y_{1} \in \mathscr{N}_{r_{1}}, y_{2} \in \mathscr{N}_{r_{1}}} R\left(\left|y_{1}\right|+\left|y_{2}\right|\right)$.

С учетом этих оценок

$$
\left\|J\left(t, t_{0}, y_{\theta}\right)\right\| \leqslant e^{\eta_{5} M^{\mu-1}}\left(1+\eta_{3}^{\prime}\left(\gamma_{2} R\left(y_{\theta}\right)\right)^{\mu-1}\left(t-t_{0}\right)\right)^{\frac{\eta_{4} \theta_{1}+\eta_{5}}{\theta_{1} \eta_{3}^{\prime}}} .
$$

Вследствие квазиоднородности функции $W(y)$ справедлива оценка

$$
\left\|\frac{\partial W}{\partial y}(y)\right\| \leqslant \eta_{6}\left\|R^{m I-G}(y)\right\|, \quad \eta_{6}=\sup _{y \in \partial \mathscr{N}_{1}}\left\|\frac{\partial W}{\partial y}\right\| .
$$

Поэтому из представления (3.12) следует оценка

$$
\begin{aligned}
& \left|W\left(y\left(s ; t_{0}, y_{2}\right)\right)-W\left(y\left(s ; t_{0}, y_{1}\right)\right)\right| \\
& \leqslant \int_{0}^{1} \| \frac{\partial W}{\partial y}\left(y\left(s ; t_{0}, y_{\theta}\right)\|\| \frac{\partial y}{\partial y_{0}}\left(s ; t_{0}, y_{\theta}\right)\|d \theta\| y_{2}-y_{1} \|\right. \\
& \leqslant \eta_{6} \int_{0}^{1}\left\|\left(\gamma_{2} R\left(y_{\theta}\right)\right)^{m I-G}\right\| \\
& \quad \times\left\|\left(1+\eta_{3}^{\prime}\left(\gamma_{2} R\left(y_{\theta}\right)\right)^{\mu-1}\left(s-t_{0}\right)\right)^{\frac{\eta_{4} \theta_{1}+\eta_{5}}{\theta_{1} \eta_{3}^{\prime}} I+\frac{m I-G}{1-\mu}}\right\| d \theta\left\|y_{2}-y_{1}\right\| \\
& \leqslant \eta_{6}^{\prime} \int_{0}^{1}\left\|R^{m I-G}\left(y_{\theta}\right)\right\| \\
& \quad \times\left\|\left(1+\eta_{3}^{\prime}\left(\gamma_{2} R\left(y_{\theta}\right)\right)^{\mu-1}\left(s-t_{0}\right)\right)^{\frac{\eta_{4} \theta_{1}+\eta_{5}}{\theta_{1} \eta_{3}^{\prime}} I+\frac{m I-G}{1-\mu}}\right\| d \theta\left\|y_{2}-y_{1}\right\| .
\end{aligned}
$$

Здесь $\eta_{6}^{\prime}=\eta_{6}\left\|\gamma_{2}^{m I-G}\right\|-$ положительная постоянная.

При $s \geqslant t_{0}$ справедлива оценка

$$
\begin{aligned}
\|(1+ & \left.\eta_{3}^{\prime}\left(\gamma_{2} R\left(y_{\theta}\right)\right)^{\mu-1}\left(s-t_{0}\right)\right)^{\frac{\eta_{4} \theta_{1}+\eta_{5}}{\theta_{1} \eta_{3}^{\prime}} I+\frac{m I-G}{1-\mu}} \| \\
& \leqslant\left(1+\eta_{3}^{\prime}\left(\gamma_{2} R\left(y_{\theta}\right)\right)^{\mu-1}\left(s-t_{0}\right)\right)^{\frac{\eta_{4} \theta_{1}+\eta_{5}}{\theta_{1} \eta_{3}^{\prime}}+\frac{m-g_{n}}{1-\mu}} .
\end{aligned}
$$

Зафиксируем положительное число $\varepsilon_{1}$ и число $m\left(m \geqslant m_{0}\right)$ выберем так, что

$$
\frac{\theta_{1} \eta_{4}+\eta_{5}}{\theta_{1} \eta_{3}^{\prime}}+\frac{m-g_{n}}{1-\mu} \leqslant 1+\varepsilon_{1} \text {. }
$$


Тогда из оценок (3.15) и (3.16) следует неравенство

$$
\begin{aligned}
\int_{t_{0}}^{+\infty} & \left|W\left(y\left(s ; t_{0}, y_{2}\right)\right)-W\left(y\left(s ; t_{0}, y_{1}\right)\right)\right| d s \\
& \leqslant \int_{t_{0}}^{+\infty} \eta_{6}^{\prime} \int_{0}^{1}\left\|R^{m I-G}\left(y_{\theta}\right)\right\|\left(1+\eta_{3}^{\prime}\left(\gamma_{2} R\left(y_{\theta}\right)\right)^{\mu-1}\left(s-t_{0}\right)\right)^{-1-\varepsilon_{1}} d \theta\left\|y_{2}-y_{1}\right\| d s \\
& =\eta_{6}^{\prime} \int_{0}^{1}\left\|R^{m I-G}\left(y_{\theta}\right)\right\| \int_{t_{0}}^{+\infty}\left(1+\eta_{3}^{\prime}\left(\gamma_{2} R\left(y_{\theta}\right)^{\mu-1}\left(s-t_{0}\right)\right)\right)^{-1-\varepsilon_{1}} d s d \theta\left\|y_{2}-y_{1}\right\| \\
& =\frac{\eta_{6}^{\prime}}{\eta_{3}^{\prime} \varepsilon_{1}} \int_{0}^{1}\left\|R^{m I-G}\left(y_{\theta}\right)\right\|\left(\gamma_{2} R\left(y_{\theta}\right)\right)^{1-\mu} d \theta\left\|y_{2}-y_{1}\right\| \\
& \leqslant \frac{\eta_{6}^{\prime} \gamma_{2}^{1-\mu}}{\eta_{3}^{\prime} \varepsilon_{1}}\left\|R^{(m+1-\mu) I-G}\left(\left|y_{1}\right|+\left|y_{2}\right|\right)\right\|\left\|y_{2}-y_{1}\right\| .
\end{aligned}
$$

Далее, не умаляя общности, предположим, что $y_{\theta} \neq 0$ при всех $\theta \in[0,1]$, и рассмотрим разность

$$
\begin{aligned}
& \left|R^{m+1-\mu}\left(y\left(s ; t_{0}, y_{2}\right)\right)-R^{m+1-\mu}\left(y\left(s ; t_{0}, y_{1}\right)\right)\right| \\
& \leqslant \int_{0}^{1}(m+1-\mu) R^{m-\mu}\left(y\left(s ; t_{0}, y_{\theta}\right)\right) \\
& \quad \times\left\|D_{y}^{+} R\left(y\left(s ; t_{0}, y_{\theta}\right)\right)\right\|\left\|J\left(s ; t_{0}, y_{\theta}\right)\right\| d \theta\left\|y_{2}-y_{1}\right\| .
\end{aligned}
$$

Очевидно, что существует положительная постоянная $\eta_{7}$ такая, что

$$
\left\|D_{y}^{+} R(y)\right\| \leqslant \eta_{7}\left\|R^{I-G}(y)\right\| .
$$

Здесь $D_{y}^{+}-$верхняя производная Дини.

\section{Поэтому}

$$
\begin{aligned}
& \left|R^{m+1-\mu}\left(y\left(s ; t_{0}, y_{2}\right)\right)-R^{m+1-\mu}\left(y\left(s ; t_{0}, y_{1}\right)\right)\right| \\
& \leqslant \eta_{7}\left\|\gamma_{2}^{(m+1-\mu) I-G}\right\|(m+1-\mu) \int_{0}^{1}\left\|R^{(m+1-\mu) I-G}\left(y_{\theta}\right)\right\| \\
& \quad \times\left\|\left(1+\eta_{3}^{\prime}\left(\gamma_{2} R\left(y_{\theta}\right)\right)^{\mu-1}\left(s-t_{0}\right)\right)^{\frac{\eta_{4} \theta_{1}+\eta_{5}}{\theta_{1} \eta_{3}^{\prime}} I+\frac{(m+1-\mu) I-G}{1-\mu}}\right\| d \theta\left\|y_{2}-y_{1}\right\| .
\end{aligned}
$$

Если это необходимо, число $m$ увеличим настолько, чтобы выполнялось неравенство

$$
\frac{\eta_{4} \theta_{1}+\eta_{5}}{\theta_{1} \eta_{3}^{\prime}}+\frac{m+1-\mu-g_{n}}{1-\mu}<0,
$$

тогда

$$
\begin{aligned}
& \sup _{s \in\left[t_{0},+\infty\right)}\left|R^{m+1-\mu}\left(y\left(s ; t_{0}, y_{2}\right)\right)-R^{m+1-\mu}\left(y\left(s ; t_{0}, y_{1}\right)\right)\right| \\
& \quad \leqslant \eta_{7}\left\|\gamma_{2}^{(m+1-\mu) I-G}\right\|(m+1-\mu)\left\|R^{(m+1-\mu) I-G}\left(\left|y_{1}\right|+\left|y_{2}\right|\right)\right\|\left\|y_{2}-y_{1}\right\| .
\end{aligned}
$$

Таким образом, теорема полностью доказана. 


\section{§ 4. Доказательство теоремы об устойчивости по нелинейному приближению}

Доказательство теоремы проведем по следующему плану: используя функцию Ляпунова для невозмущенной системы (2.2), существование которой доказано в теореме 2, установим оценки полной производной и полной разности этой функции вдоль решений возмущенной системы (2.1). На основе этих оценок установим устойчивость и асимптотическую устойчивость решения $x=0$ системы (2.1).

Для исследования устойчивости состояния равновесия $x=0$ системы $(2.1)$ применим функцию Ляпунова $V(t, x)$, существование которой обеспечивает теорема 2 .

Предварительно установим оценки для вспомогательной функции $V(t, x)$ вдоль решений системы дифференциальных уравнений (2.1) в достаточно малой окрестности $\mathscr{N}_{r_{0}}, r_{0}>0$, состояния равновесия $x=0$.

Пусть $\varepsilon>0$, тогда

$$
\begin{gathered}
\frac{V(t+\varepsilon, x+\varepsilon f(x)+\varepsilon P(t, x))-V(t, x)}{\varepsilon}=\frac{V(t+\varepsilon, x+\varepsilon f(x))-V(t, x)}{\varepsilon} \\
\quad+\frac{V(t+\varepsilon, x+\varepsilon f(x)+\varepsilon P(t, x))-V(t+\varepsilon, x+\varepsilon f(x))}{\varepsilon} \\
\leqslant \frac{V(t+\varepsilon, x+\varepsilon f(x))-V(t, x)}{\varepsilon} \\
+\gamma_{3}\left\|R^{(m-\mu+1) I-G}(2 x+2 \varepsilon f(x)+\varepsilon P(t, x))\right\|\|P(t, x)\| .
\end{gathered}
$$

Переходя к пределу при $\varepsilon \rightarrow 0+$, получим неравенство

$$
\begin{aligned}
\left.D^{+} V(t, x)\right|_{(2.1)} & \leqslant\left. D^{+} V(t, x)\right|_{(2.2)}+\gamma_{3}\left\|R^{(m-\mu+1) I-G}(2 x)\right\|\|P(t, x)\| \\
& \leqslant-W(x)+\gamma_{3}\left\|R^{(m-\mu+1) I-G}(2 x)\right\|\|P(t, x)\| .
\end{aligned}
$$

В достаточно малой окрестности $\mathscr{N}_{r_{0}}$ состояния равновесия $x=0$ для некоторой положительной постоянной $C_{1}$ справедливо неравенство

$$
\frac{\left\|R^{(m-\mu+1) I-G}(2 x)\right\|}{\left\|R^{(m-\mu+1) I-G}(x)\right\|} \leqslant C_{1} .
$$

Поэтому в этой окрестности справедливо неравенство

$$
\begin{aligned}
\left.D^{+} V(t, x)\right|_{(2.1)} & \leqslant-\eta_{1} R^{m}(x)+\gamma_{3} C_{1}\left\|R^{(m-\mu+1) I-G}(x)\right\|\left\|R^{(\mu-1) I+G}(x)\right\| \psi_{1}(x) \\
& \leqslant\left(-\eta_{1}+\gamma_{3} C_{1} \psi_{1}(x)\right) R^{m}(x)
\end{aligned}
$$

где $\psi_{1}(x)$ - положительная непрерывная функция, $\psi_{1}(x) \rightarrow 0$ при $\|x\| \rightarrow 0$. Уменьшая, если это необходимо, окрестность состояния равновесия $x=0$, можно добиться выполнения неравенства

$$
\psi_{1}(x) \leqslant \frac{\eta_{1}}{2 \gamma_{3} C_{1}}
$$

тогда

$$
\left.D^{+} V(t, x)\right|_{(2.1)} \leqslant-\frac{\eta_{1}}{2} R^{m}(x)
$$


Оценка разности вспомогательной функции $V(t, x)$ вдоль решений нелинейной системы (2.1) при $t=\tau_{k}$ имеет вид

$$
\begin{aligned}
& V\left(t+0, x+g(x)+Q_{k}(x)\right)-V(t, x) \\
& \quad \leqslant \gamma_{3}\left\|R^{(m-\mu+1) I-G}\left(2 x+2 g(x)+Q_{k}(x)\right) R^{(\mu-1) I+G}(x)\right\| \psi_{2}(x),
\end{aligned}
$$

где $\psi_{2}(x)$ - непрерывная положительная функция, $\psi_{2}(x) \rightarrow 0$ при $\|x\| \rightarrow 0$.

Отметим, что существует положительная постоянная $\eta_{8}$ такая, что в достаточно малой окрестности $\mathscr{N}_{r_{0}}\left(r_{0}\right.$, возможно, необходимо уменьшить) состояния равновесия $x=0$ выполняется неравенство

$$
\Phi(x) \equiv\left\|R^{(m-\mu+1) I-G}\left(2 x+2 g(x)+Q_{k}(x)\right) R^{(\mu-1) I+G}(x)\right\| \leqslant \eta_{8} R^{m}(x) .
$$

Докажем это утверждение. Очевидно, что

$$
\Phi(x)=\alpha^{m}\left\|R^{(m-\mu+1) I-G}\left(2 \alpha^{-G} x+2 \alpha^{-G} g(x)+\alpha^{-G} Q_{k}(x)\right) R^{(\mu-1) I+G}\left(\alpha^{-G} x\right)\right\| .
$$

Пусть $\alpha=R(x)$, тогда $R\left(\alpha^{-G} x\right)=1$ и, как следствие, при всех $x \in \mathbb{R}^{n}$ выполняется неравенство $\left\|\alpha^{-G} x\right\| \leqslant r^{*}$, где $r^{*}$ - положительная постоянная. Из очевидного тождества $\alpha^{-G} g(x)=\alpha^{\mu-1} g\left(\alpha^{-G} x\right)$ следует неравенство

$$
\left\|\alpha^{-G} g(x)\right\| \leqslant \eta_{9} R^{\mu-1}(x),
$$

где $\eta_{9}=\sup _{x \in \partial \mathscr{N}_{1}}\|g(x)\|$.

Непосредственно из условия теоремы следует, что

$$
\left\|\alpha^{-G} Q_{k}(x)\right\| \leqslant R^{\mu-1}(x) \psi_{2}(x) .
$$

Обозначим через $r^{* *}$ максимум функции $2 r^{*}+2 \eta_{9} R^{\mu-1}(x)+R^{\mu-1}(x) \psi_{2}(x)$ в рассматриваемой окрестности состояния равновесия $x=0$ и обозначим $\eta_{8}=$ $\sup _{\|x\| \leqslant r^{* *}}\left\|R^{(m-\mu+1) I-G}(x)\right\|$, тогда получим требуемую оценку.

Таким образом,

$$
V\left(t+0, x+g(x)+Q_{k}(x)\right)-V(t, x) \leqslant \gamma_{3} \eta_{8} R^{m}(x) \psi_{2}(x)
$$

и существует достаточно малое положительное число $r_{0}$ такое, что в окрестности $\mathscr{N}_{r_{0}}$ выполняются оценки (4.1) и (4.2).

Покажем, что можно выбрать положительное число $r_{1}<r_{0}$ и соответствующее положительное число $\delta_{1}$ настолько малым, что любое решение $x\left(t ; t_{0}, x_{0}\right)$ с начальными условиями $\left\|x_{0}\right\|<\delta_{1}$ не выходит за пределы окрестности $\mathscr{N}_{r_{1}}$ при всех $t \geqslant t_{0}$. Число $r_{1}$ выберем так, чтобы $r_{1}<r_{0}$, и зафиксируем число $t^{*}>\tau_{2}$. Тогда вследствие непрерывной зависимости решений задачи Коши $x\left(t ; t_{0}, x_{0}\right)$ от начального условия $x_{0}$ существует число $\delta_{1}>0$ такое, что $x\left(t ; t_{0}, x_{0}\right) \in \mathscr{N}_{r_{1}}$ при всех $t \in\left[t_{0}, t^{*}\right), x\left(t^{*} ; t_{0}, x_{0}\right) \notin \mathscr{N}_{r_{1}}$. Обозначим через $k_{0}$ наибольшее натуральное число, для которого $\tau_{k_{0}}<t^{*}$. Тогда

$$
\begin{aligned}
V\left(t^{*}, x\left(t^{*} ; t_{0}, x_{0}\right)\right)-V\left(\tau_{1}+0, x\left(\tau_{1}+0 ; t_{0}, x_{0}\right)\right) \leqslant-\frac{\eta_{1}}{2} \int_{\tau_{k_{0}}}^{t^{*}} R^{m}\left(x\left(s ; t_{0}, x_{0}\right)\right) d s \\
\quad-\frac{\eta_{1}}{2} \sum_{k=2}^{k_{0}} \int_{\tau_{k-1}}^{\tau_{k}} R^{m}\left(x\left(s ; t_{0}, x_{0}\right)\right) d s-\frac{\eta_{1}}{2} \int_{t_{0}}^{\tau_{1}} R^{m}\left(x\left(s ; t_{0}, x_{0}\right)\right) d s \\
\quad+\sum_{k=2}^{k_{0}} \gamma_{3} \eta_{8} R^{m}\left(x\left(\tau_{k} ; t_{0}, x_{0}\right)\right) \psi_{2}\left(x\left(\tau_{k} ; t_{0}, x_{0}\right)\right) .
\end{aligned}
$$


Нетрудно показать, что существует положительная постоянная $\eta_{10}$ такая, что

$$
\left|\frac{d R\left(x\left(t ; t_{0}, x_{0}\right)\right)}{d t}\right| \leqslant \eta_{10} R^{\mu}\left(x\left(t ; t_{0}, x_{0}\right)\right) .
$$

Как следствие, $s \in\left(\tau_{k-1}, \tau_{k}\right]$ и

$$
R^{1-\mu}\left(x\left(s ; t_{0}, x_{0}\right)\right)-R^{1-\mu}\left(x\left(\tau_{k} ; t_{0}, x_{0}\right)\right) \leqslant \eta_{10}(\mu-1)\left(\tau_{k}-s\right) \leqslant \eta_{10}(\mu-1) \theta_{2} .
$$

Отсюда находим

$$
\begin{aligned}
R\left(x\left(s ; t_{0}, x_{0}\right)\right) & \geqslant R\left(x\left(\tau_{k} ; t_{0}, x_{0}\right)\right)\left(1+\eta_{10}(\mu-1) \theta_{2} R^{\mu-1}\left(x\left(\tau_{k} ; t_{0}, x_{0}\right)\right)\right)^{\frac{1}{1-\mu}} \\
& \geqslant R\left(x\left(\tau_{k} ; t_{0}, x_{0}\right)\right)\left(1+\eta_{10}(\mu-1) \theta_{2} r_{0}^{\mu-1}\right)^{\frac{1}{1-\mu}} \equiv \eta_{11} R\left(x\left(\tau_{k} ; t_{0}, x_{0}\right)\right) .
\end{aligned}
$$

Также из неравенства (4.3) следует оценка

$$
R\left(x\left(\tau_{1} ; t_{0}, x_{0}\right)\right) \leqslant R\left(x_{0}\right)\left(1-\eta_{10}(\mu-1) R^{\mu-1}\left(x_{0}\right)\left(\tau_{1}-t_{0}\right)\right)^{\frac{1}{1-\mu}},
$$

если только выполняется неравенство $1-\eta_{10}(\mu-1) R^{\mu-1}\left(x_{0}\right)\left(\tau_{1}-t_{0}\right)>0$.

Аналогично, справедлива оценка

$$
\begin{aligned}
R\left(x\left(\tau_{k} ; t_{0}, x_{0}\right)\right) & \leqslant R\left(x\left(\tau_{k-1}+0 ; t_{0}, x_{0}\right)\right) \\
& \times\left(1-\eta_{10}(\mu-1) R^{\mu-1}\left(x\left(\tau_{k-1}+0 ; t_{0}, x_{0}\right)\right)\left(\tau_{k}-\tau_{k-1}\right)\right)^{\frac{1}{1-\mu}}
\end{aligned}
$$

если только выполняется неравенство

$$
1-\eta_{10}(\mu-1) R^{\mu-1}\left(x\left(\tau_{k-1}+0 ; t_{0}, x_{0}\right)\right)\left(\tau_{k}-\tau_{k-1}\right)>0 .
$$

Поэтому справедлива оценка

$$
\begin{aligned}
& V\left(t^{*}, x\left(t^{*} ; t_{0}, x_{0}\right)\right)-V\left(\tau_{1}+0, x\left(\tau_{1}+0 ; t_{0}, x_{0}\right)\right) \\
& \quad \leqslant \sum_{k=2}^{k_{0}}\left(-\frac{\eta_{1} \eta_{11}^{m}}{2}\left(\tau_{k}-\tau_{k-1}\right)+\gamma_{3} \eta_{8} \psi_{2}\left(x\left(\tau_{k} ; t_{0}, x_{0}\right)\right)\right) R^{m}\left(x\left(\tau_{k} ; t_{0}, x_{0}\right)\right) .
\end{aligned}
$$

Число $r_{1}$ выберем настолько малым, чтобы в окрестности $\mathscr{N}_{r_{1}}$ точки $x=0$ выполнялось неравенство

$$
\psi_{2}(x) \leqslant \frac{\eta_{1} \eta_{11}^{m} \theta_{1}}{4 \gamma_{3} \eta_{8}}
$$

тогда

$$
V\left(t^{*}, x\left(t^{*} ; t_{0}, x_{0}\right)\right)-V\left(\tau_{1}+0, x\left(\tau_{1}+0 ; t_{0}, x_{0}\right)\right) \leqslant-\frac{\eta_{1} \eta_{11}^{m} \theta_{1}}{4} \sum_{k=2}^{k_{0}} R^{m}\left(x\left(\tau_{k} ; t_{0}, x_{0}\right)\right) .
$$

Число $\delta_{1}>0$ выберем настолько малым, чтобы при всех $x_{0}$ таких, что $\left\|x_{0}\right\|<\delta_{1}$, выполнялось неравенство

$$
\begin{aligned}
& R\left(x_{0}\right)<\min {\left[\left(\frac{1}{2 \eta_{10}(\mu-1)\left(\tau_{1}-t_{0}\right)}\right)^{\frac{1}{\mu-1}},\right.} \\
&\left.r_{1}\left(\frac{\gamma_{2}}{\gamma_{1}}\left(1+\frac{\gamma_{3} \eta_{8} 2^{\frac{m}{\mu-1}} r_{0}^{\mu-1}}{\gamma_{2}}\right)\right)^{-\frac{1}{m+1-\mu}}\right] .
\end{aligned}
$$


Тогда справедливо неравенство $R\left(x\left(\tau_{1} ; t_{0}, x_{0}\right)\right) \leqslant 2^{1 /(\mu-1)} R\left(x_{0}\right)$ и, как следствие, неравенство

$$
\begin{array}{r}
V\left(t^{*}, x\left(t^{*} ; t_{0}, x_{0}\right)\right) \leqslant \gamma_{2} R^{m-\mu+1}\left(x_{0}\right)+\gamma_{3} \eta_{8} 2^{\frac{m}{\mu-1}} R^{m}\left(x_{0}\right) \\
-\frac{\eta_{1} \eta_{11}^{m} \theta_{1}}{4} \sum_{k=2}^{k_{0}} R^{m}\left(x\left(\tau_{k} ; t_{0}, x_{0}\right)\right) .
\end{array}
$$

Из оценок (3.4) для функции Ляпунова $V(t, x)$ следует, что

$$
\begin{aligned}
\gamma_{1} R^{m+1-\mu}\left(x\left(t^{*} ; t_{0}, x_{0}\right)\right) & \leqslant \gamma_{2} R^{m+1-\mu}\left(x_{0}\right)+\gamma_{3} \eta_{8} 2^{\frac{m}{\mu-1}} R^{m}\left(x_{0}\right) \\
& \leqslant \gamma_{2} R^{m+1-\mu}\left(x_{0}\right)\left(1+\frac{\gamma_{3} \eta_{8} 2^{\frac{m}{\mu-1}} r_{0}^{\mu-1}}{\gamma_{2}}\right) .
\end{aligned}
$$

Отсюда находим

$$
R\left(x\left(t^{*} ; t_{0}, x_{0}\right)\right) \leqslant R\left(x_{0}\right)\left(\frac{\gamma_{2}}{\gamma_{1}}\left(1+\frac{\gamma_{3} \eta_{8} 2^{\frac{m}{\mu-1}} r_{0}^{\mu-1}}{\gamma_{2}}\right)\right)^{\frac{1}{m+1-\mu}}
$$

Тогда

$$
r_{1} \leqslant R\left(x\left(t^{*} ; t_{0}, x_{0}\right)\right)<r_{1} .
$$

Полученное противоречие доказывает, что при всех $t \geqslant t_{0}$ решение $x\left(t ; t_{0}, x_{0}\right)$ не покидает окрестность $\mathscr{N}_{r_{1}}$ точки $x=0$. Поэтому при всех $t>\tau_{2}$ выполняется неравенство

$$
V\left(t, x\left(t ; t_{0}, x_{0}\right)\right) \leqslant\left\{\begin{aligned}
\gamma_{2} R^{m-\mu+1}\left(x_{0}\right)+\gamma_{3} \eta_{8} 2^{\frac{m}{\mu-1}} R^{m}\left(x_{0}\right) & \\
-\frac{\eta_{1} \eta_{11}^{m} \theta_{1}}{4} \sum_{k=2}^{k_{0}} R^{m}\left(x\left(\tau_{k} ; t_{0}, x_{0}\right)\right), & t>\tau_{2}, \\
\gamma_{2} R^{m-\mu+1}\left(x_{0}\right)+\gamma_{3} \eta_{8} 2^{\frac{m}{\mu-1}} R^{m}\left(x_{0}\right), \quad & t \in\left(\tau_{1}, \tau_{2}\right] .
\end{aligned}\right.
$$

Из этих рассуждений также следует, что все решения $x\left(t ; t_{0}, x_{0}\right)$ бесконечно продолжимы на интервал $\left[t_{0},+\infty\right)$ (см. теорему 2.1 из [15]). Неравенство (4.4) позволяет доказать устойчивость состояния равновесия $x=0$ системы (2.1).

Возьмем $\varepsilon>0$ и зададим положительное число $\delta, \delta<\delta_{1}$, так, что

$$
\delta<\varepsilon^{\frac{1}{g_{s}}}\left(\frac{\gamma_{2}}{\gamma_{1}}\left(1+\frac{\gamma_{3} \eta_{8} 2^{\frac{m}{\mu-1}} r_{0}^{\mu-1}}{\gamma_{2}}\right)\right)^{\frac{1}{m+1-\mu}}, \quad s=\overline{1, n} .
$$

Тогда $R\left(x\left(t ; t_{0}, x_{0}\right)\right)<\varepsilon^{\frac{1}{g_{s}}}$ при всех $t \geqslant t_{0}$, поэтому $\left|x_{s}\left(t ; t_{0}, x_{0}\right)\right|<\varepsilon$, что доказывает устойчивость состояния равновесия $x=0$ системы $(2.1)$.

Установим асимптотическую устойчивость состояния равновесия $x=0$. Вследствие доказанного выше свойства устойчивости решения $x=0$ системы (2.1) можно выбрать положительное число $\rho_{0}$ такое, что из неравенства $\left\|x_{0}\right\|<\rho_{0}$ следует, что $R\left(x\left(t ; t_{0}, x_{0}\right)\right)<\left(2 \theta_{2}(\mu-1) \eta_{10}\right)^{\frac{1}{1-\mu}}$ при всех $t \geqslant t_{0}$.

Последовательность чисел $\left\{V\left(\tau_{k}, x\left(\tau_{k} ; t_{0}, x_{0}\right)\right)\right\}_{k=1}^{\infty}$ не возрастает, поэтому существует число $\alpha \geqslant 0$ такое, что

$$
\alpha=\lim _{k \rightarrow \infty} V\left(\tau_{k}, x\left(\tau_{k} ; t_{0}, x_{0}\right)\right)=\inf _{k} V\left(\tau_{k}, x\left(\tau_{k} ; t_{0}, x_{0}\right)\right) .
$$

Предположим, от противного, что $\alpha>0$, тогда при всех $k \in \mathbb{N}$

$$
V\left(\tau_{k}, x\left(\tau_{k} ; t_{0}, x_{0}\right)\right) \geqslant \alpha>0 .
$$


Отметим, что $\inf _{k} R\left(x\left(\tau_{k} ; t_{0}, x_{0}\right)\right)=\beta>0$. Действительно, в противном случае существует подпоследовательность $\left\{R\left(x\left(\tau_{n_{k}} ; t_{0}, x_{0}\right)\right)\right\}_{k=1}^{\infty}$ последовательности $\left\{R\left(x\left(\tau_{k} ; t_{0}, x_{0}\right)\right)\right\}_{k=1}^{\infty}$, сходящаяся к нулю при $k \rightarrow+\infty$. Из свойства функции $V(t, x)$ следует неравенство

$$
0<\alpha \leqslant V\left(\tau_{n_{k}}, x\left(\tau_{n_{k}} ; t_{0}, x_{0}\right)\right) \leqslant \gamma_{2} R^{m-\mu+1}\left(x\left(\tau_{n_{k}} ; t_{0}, x_{0}\right)\right) .
$$

При $k \rightarrow \infty$ получим противоречие, и, как следствие, $R\left(x\left(\tau_{k} ; t_{0}, x_{0}\right)\right) \geqslant \beta$ при всех $k \in \mathbb{N}$. Таким образом, с учетом неравенства (4.4) получим при $k \geqslant 2$ оценку

$$
\alpha \leqslant V\left(\tau_{k}, x\left(\tau_{k} ; t_{0}, x_{0}\right)\right) \leqslant V\left(\tau_{1}+0, x\left(\tau_{1}+0 ; t_{0}, x_{0}\right)\right)-(k-1) \frac{\eta_{1} \eta_{11}^{m} \theta_{1} \beta^{m}}{4} .
$$

При достаточно большом $k$ получим противоречие, которое доказывает, что $\lim _{k \rightarrow \infty} V\left(\tau_{k}, x\left(\tau_{k} ; t_{0}, x_{0}\right)\right)=0$. Обозначим $\gamma=\limsup _{k \rightarrow \infty} R\left(x\left(\tau_{k} ; t_{0}, x_{0}\right)\right) \geqslant 0$, тогда существует подпоследовательность $\left\{R\left(x\left(\tau_{n_{k}} ; t_{0}, x_{0}\right)\right)\right\}_{k=1}^{\infty}$ последовательности $\left\{R\left(x\left(\tau_{k} ; t_{0}, x_{0}\right)\right)\right\}_{k=1}^{\infty}$, сходящаяся к $\gamma$ при $k \rightarrow+\infty$. Из неравенства

$$
\gamma_{1} R^{m-\mu+1}\left(x\left(\tau_{n_{k}} ; t_{0}, x_{0}\right)\right) \leqslant V\left(\tau_{n_{k}}, x\left(\tau_{n_{k}} ; t_{0}, x_{0}\right)\right)
$$

переходом к пределу $k \rightarrow \infty$ получим $\gamma=0$.

Поэтому $x\left(\tau_{k} ; t_{0}, x_{0}\right) \rightarrow 0$ при $k \rightarrow \infty$, и из неравенства

$$
R\left(x\left(t ; t_{0}, x_{0}\right)\right) \leqslant 2^{\frac{1}{\mu-1}} R\left(x\left(\tau_{k-1}+0 ; t_{0}, x_{0}\right)\right)
$$

следует, что $x\left(t ; t_{0}, x_{0}\right) \rightarrow 0$ при $t \rightarrow \infty$. Теорема доказана.

\section{§5. Априорные оценки решений}

Конструктивное применение теоремы 1 сопряжено не только с необходимостью дать ответ на вопрос об асимптотической устойчивости состояния равновесия $y=0$ исходной системы (2.2), но и также с необходимостью доказать априорные оценки вида (2.4) для семейства нелинейных систем (2.3). В настоящем разделе, развивая идеи работ [22], [23], укажем два класса систем дифференциальных уравнений, для которых получение такого рода оценок представляется возможным.

Предварительно сформулируем в виде леммы один результат, касающийся нелинейных разностных уравнений, заимствованный из $[24 ;$ ч. 1 , отд. 1 , гл. 1 , $\S 2$, задача 174].

Лемма 3. Пусть в интервале $0<x<x_{0}$ имеем $0<\vartheta(x)<x$,

$$
\vartheta(x)=x-a x^{k}+b x^{l}+x^{l} \epsilon(x), \quad \lim _{x \rightarrow 0} \epsilon(x)=0,
$$

причем $1<k<l$, а и $b$ - положительные числа. Полагаем

$$
\nu_{0}=x, \quad \nu_{1}=\vartheta\left(\nu_{0}\right), \quad \nu_{1}=\vartheta\left(\nu_{0}\right), \quad \nu_{2}=\vartheta\left(\nu_{1}\right), \quad \ldots, \quad \nu_{n}=\vartheta\left(\nu_{n-1}\right), \quad \ldots
$$

Тогда

$$
\lim _{n \rightarrow \infty} n^{\frac{1}{k-1}} \nu_{n}=[(k-1) a]^{\frac{1}{1-k}} .
$$

Доказательство приведено в [24; ч. 2]. 
5.1. Квазиоднородные системы Важевского. Предположим, что для (2.3) существует вектор-функция Ляпунова $V(t, z)=\left(V_{1}(t, z), \ldots, V_{s}(t, z)\right)^{T}$, не зависящая от параметра $\varepsilon \in(0,1]$ и удовлетворяющая в достаточно малой окрестности состояния равновесия $z=0$ системы (2.3) следующим условиям.

1. Функции $V_{i}(t, z), i=\overline{1, s}$, положительно полуопределенные, и существуют положительные постоянные $\underline{\gamma}, \varkappa$ такие, что

$$
\underline{\gamma}\|z\|^{\varkappa} \leqslant \sum_{i=1}^{s} V_{i}(t, z) .
$$

2. Выполняются оценки при всех $i=\overline{1, s}$

$$
\begin{gathered}
\left.D^{+} V_{i}(t, z)\right|_{(2.3)} \leqslant \widehat{f}_{i}\left(V_{1}(t, z), \ldots, V_{s}(t, z)\right), \quad t \neq \varepsilon^{\mu-1} \tau_{k}, \\
V_{i}\left(t+0, z+\varepsilon^{\mu-1} g(z)\right)-V_{i}(t, z) \leqslant \varepsilon^{\mu-1} \widehat{g}_{i}\left(V_{1}(t, z), \ldots, V_{s}(t, z)\right), \quad t=\varepsilon^{\mu-1} \tau_{k} .
\end{gathered}
$$

Если $\widehat{f}(u)$ удовлетворяет условиям Важевского, а функция $u+\varepsilon^{\mu-1} \widehat{g}(u)$ является неубывающей по $u$ (в некоторой окрестности $\mathscr{N} \subset \mathbb{R}^{s}$ точки $u=0$ ) при всех $\varepsilon \in(0,1]$, то лемма 1 гарантирует выполнение неравенств

$$
V_{i}\left(t, z\left(t ; t_{0}, z_{0}\right)\right) \leqslant u_{i}\left(t ; V_{1}\left(t_{0}, z_{0}\right), \ldots, V_{s}\left(t_{0}, z_{0}\right), \varepsilon\right),
$$

где $u_{i}\left(t ; t_{0}, u_{0}, \varepsilon\right), i=\overline{1, s},-$ решение задачи Коши для системы сравнения

$$
\begin{gathered}
\frac{d u}{d t}=\widehat{f}(u), \quad t \neq \varepsilon^{\mu-1} \tau_{k}, \\
\Delta u(t)=\varepsilon^{\mu-1} \widehat{g}(u(t)), \quad t=\varepsilon^{\mu-1} \tau_{k} .
\end{gathered}
$$

В силу свойства 1 и неравенства (5.1) достаточно рассмотреть вопрос об априорных (равномерных по параметру $\varepsilon \in(0,1])$ оценках решений семейства нелинейных систем (2.3). Далее ограничимся случаем, когда функции $\widehat{f}$ и $\widehat{g}$ являются квазиднородными функциями (в конусе $\mathbb{R}_{+}^{s}$ ) измерения $q$ типа $K=$ $\operatorname{diag}\left(k_{1}, \ldots, k_{s}\right)$, т.е. при всех $\alpha \geqslant 0, u \in \mathbb{R}_{+}^{s}$ выполняются равенства

$$
\widehat{f}\left(\alpha^{K} u\right)=\alpha^{K+(q-1) I} \widehat{f}(u), \quad \widehat{g}\left(\alpha^{K} u\right)=\alpha^{K+(q-1) I} \widehat{g}(u) .
$$

ТЕОрема 3. Предположим, что $K \geqslant I$ и существует вектор $w>0$ такой, что выполняются неравенства

$$
\begin{gathered}
\widehat{f}(w) \leqslant \gamma_{0} K w, \quad \widehat{g}(w) \leqslant \beta_{0} K w, \\
\sup _{k}\left(\gamma_{0}\left(\tau_{k+1}-\tau_{k}\right)+\beta_{0}\right)<0 .
\end{gathered}
$$

Тогда для решений однопараметрического семейства систем сравнения (5.2) выполняется равномерно по параметру $\varepsilon \in(0,1]$ априорная оченка вида $(2.4)$.

ДокАзАтЕльство. Обозначим через $\psi\left(t ; t_{0}, \psi_{0}\right)$ решение задачи Коши для дифференциального уравнения сравнения

$$
\begin{gathered}
\frac{d \psi}{d t}=\gamma_{0} \psi^{q}(t), \quad t \neq \varepsilon^{q-1} \tau_{k}, \\
\Delta \psi(t)=\varepsilon^{q-1} \beta_{0} \psi^{q}(t), \quad t=\varepsilon^{q-1} \tau_{k} .
\end{gathered}
$$


Предварительно установим априорную оценку решений для этого семейства уравнений. Введем разностное уравнение

$$
z_{k}=\left[z_{k-1}^{1-q}+\varepsilon^{q-1} \gamma_{0}\left(\tau_{k}-\tau_{k}\right)\right]^{\frac{q}{1-q}}\left[z_{k-1}^{1-q}+\left(\gamma_{0}\left(\tau_{k}-\tau_{k-1}\right)+\beta_{0}\right) \varepsilon^{q-1}\right]
$$

и замену переменных $z_{k}=\varepsilon^{-1} \widetilde{z}_{k}$. Тогда

$$
\widetilde{z}_{k}=\left[\widetilde{z}_{k-1}^{1-q}+\gamma_{0}\left(\tau_{k}-\tau_{k}\right)\right]^{\frac{q}{1-q}}\left[\widetilde{z}_{k-1}^{1-q}+\left(\gamma_{0}\left(\tau_{k}-\tau_{k-1}\right)+\beta_{0}\right)\right] \equiv \Psi_{k-1}\left(\widetilde{z}_{k-1}\right) .
$$

В достаточно малой окрестности точки $z=0$ справедливо представление

$$
\Psi_{k-1}(z)=z+\left(\gamma_{0}\left(\tau_{k}-\tau_{k-1}\right)+\beta_{0}\right) z^{q}+\widetilde{\Psi}_{k-1}(z),
$$

где $\widetilde{\Psi}_{k-1}(z) / z^{q} \rightarrow 0$ при $z \rightarrow 0$ равномерно по $k \in \mathbb{N}$. Обозначим

$$
\eta_{0}=-\sup _{k}\left(\gamma_{0}\left(\tau_{k}-\tau_{k-1}\right)+\beta_{0}\right)>0 .
$$

Покажем сначала, что существует положительное достаточно малое число $\delta_{1}$, не зависящее от $\varepsilon$, такое, что из условия $z_{1} \in\left(0, \delta_{1}\right)$ следует, что $z_{k}$ существует при всех $k \in \mathbb{N}$ и при этом $z_{k}>0$. Выберем число $\delta_{1}$ так, чтобы

$$
\delta_{1}<\frac{1}{2} \min \left\{\left(\left|\gamma_{0}\right| \theta_{2}\right)^{\frac{1}{1-q}},\left(\left|\gamma_{0}\right| \theta_{2}+\left|\beta_{0}\right|\right)^{\frac{1}{1-q}}\right\} \equiv r_{1}
$$

и $\widetilde{\Psi}_{k-1}(z) \leqslant \eta_{0} z^{q} / 2$ при всех $z \in\left(0, \delta_{1}\right), k \in \mathbb{N}$.

Обозначим через $k_{0}$ натуральное число такое, что $\widetilde{z}_{k}<r_{1}$ при всех $k, 1 \leqslant$ $k<k_{0}, \widetilde{z}_{k_{0}} \geqslant r_{1}$. Тогда

$$
r_{1} \leqslant \widetilde{z}_{k_{0}} \leqslant \widetilde{z}_{k_{0}-1}-\frac{\eta_{0}}{2} \widetilde{z}_{k_{0}-1}^{q}<r_{1}
$$

Полученное противоречие доказывает, что $\widetilde{z}_{k} \in\left(0, r_{1}\right)$ при всех $k \in \mathbb{N}$. Из леммы 3 следует, что

$$
\widetilde{z}_{k} k^{\frac{1}{q-1}} \leqslant c_{0},
$$

где $c_{0}$ - положительная постоянная. Поэтому

$$
z_{k} \leqslant \varepsilon^{-1} c_{0} k^{\frac{1}{1-q}} .
$$

Вследствие непрерывной зависимости решений скалярного уравнения $\dot{\psi}=\gamma_{0} \psi^{q}$ можно указать положительное число $\delta_{2}$ (не зависящее от $\varepsilon$ ) такое, что при всех $\psi_{0} \in\left(0, \delta_{2}\right)$ выполняется включение $\psi\left(\varepsilon^{q-1} \tau_{1}+0 ; t_{0}, \psi_{0}, \varepsilon\right) \in\left(0, \delta_{1}\right)$. В этом случае последовательность $z_{k}=\psi\left(\varepsilon^{q-1} \tau_{k}+0 ; t_{0}, \psi_{0}, \varepsilon\right)$ удовлетворяет разностному уравнению (5.4). Пусть $t \in\left(\varepsilon^{q-1} \tau_{k}, \varepsilon^{q-1} \tau_{k+1}\right]$, тогда

$$
\begin{aligned}
\psi\left(t ; t_{0}, \psi_{0}, \varepsilon\right) & =\left[z_{k}^{1-q}+\gamma_{0}\left(t-\varepsilon^{q-1} \tau_{k}\right)\right]^{\frac{1}{1-q}} \leqslant z_{k}\left[1-\left|\gamma_{0}\right|\left(r_{1} \theta_{2}\right)^{q-1}\right]^{\frac{1}{1-q}} \\
& \leqslant \varepsilon^{-1} c_{0} k^{\frac{1}{1-q}}\left[1-\left|\gamma_{0}\right|\left(r_{1} \theta_{2}\right)^{q-1}\right]^{\frac{1}{1-q}} .
\end{aligned}
$$

Поскольку $\varepsilon^{1-q}\left(t-\varepsilon^{q-1} \tau_{1}\right) \leqslant \theta_{2} k$, то из последнего неравенства следует, что

$$
\begin{aligned}
\psi\left(t ; t_{0}, \psi_{0}, \varepsilon\right) & \leqslant c_{0}\left[1-\left|\gamma_{0}\right|\left(r_{1} \theta_{2}\right)^{q-1}\right]^{\frac{1}{1-q}}\left(t-\varepsilon^{q-1} \tau_{1}\right)^{\frac{1}{1-q}} \theta_{2}^{\frac{1}{1-q}} \\
& =c_{0}\left[1-\left|\gamma_{0}\right|\left(r_{1} \theta_{2}\right)^{q-1}\right]^{\frac{1}{1-q}} \theta_{2}^{\frac{1}{1-q}}\left(t-t_{0}\right)^{\frac{1}{1-q}}\left(\frac{t-\varepsilon^{q-1} \tau_{1}}{t-t_{0}}\right)^{\frac{1}{1-q}} .
\end{aligned}
$$


Поскольку $\frac{t-\varepsilon^{q-1} \tau_{1}}{t-t_{0}} \rightarrow 1$ при $t \rightarrow+\infty$ равномерно по $\varepsilon \in(0,1)$, то существует $T \geqslant 0$, не зависящее от $\varepsilon \in(0,1]$, такое, что при всех $t \geqslant t_{0}+T$ выполняется оценка

$$
\psi\left(t ; t_{0}, \psi_{0}, \varepsilon\right) \leqslant C_{0}\left(t-t_{0}\right)^{\frac{1}{1-q}},
$$

где $C_{0}$ - положительная постоянная. Таким образом, априорная оценка установлена для уравнения сравнения (5.3).

Рассмотрим функцию $v(t)=\psi^{K}(t) w$. Тогда, используя условия теоремы и предположения относительно квазиоднородности функций $\widehat{f}(u)$ и $\widehat{g}(u)$, нетрудно установить справедливость неравенства

$$
\frac{d v}{d t} \geqslant \widehat{f}(v(t)), \quad t \neq \varepsilon^{q-1} \tau_{k},
$$

при всех $t \in\left[t_{0}, \infty\right)$, для которых решение задачи Коши $u\left(t ; t_{0}, \psi_{0}^{K} w\right)$ существует.

Рассмотрим функцию $v(t)$ при $t=\varepsilon^{q-1} \tau_{k}$

$$
\begin{aligned}
v\left(\varepsilon^{q-1} \tau_{k}+0\right) & =\psi^{K}\left(\varepsilon^{q-1} \tau_{k}+0\right) w=\left(\psi\left(\varepsilon^{q-1} \tau_{k}\right)+\beta_{0} \psi^{q}\left(\varepsilon^{q-1} \tau_{k}\right)\right)^{K} w \\
& =\psi^{K}\left(\varepsilon^{q-1} \tau_{k}\right)\left(1+\beta_{0} \psi^{q-1}\left(\varepsilon^{q-1} \tau_{k}\right)\right)^{K} w .
\end{aligned}
$$

Вследствие асимптотической устойчивости решения $\psi=0$ скалярного дифференциального уравнения сравнения (5.3) существует положительная постоянная $\delta_{3}$ такая, что при всех $\psi_{0} \in\left(0, \delta_{3}\right)$ решение задачи Коши $\psi\left(t ; t_{0}, \psi_{0}\right)$ существует при всех $t \geqslant t_{0}$ и выполняются неравенство $\psi\left(t ; t_{0}, \psi_{0}\right)<\left|\beta_{0}\right|^{\frac{1}{1-q}}$ и включение $\psi\left(t ; t_{0}, \psi_{0}\right) w \in \mathscr{N}$. Тогда вследствие неравенства Бернулли

$$
(1+x)^{K} \geqslant I+K x, \quad|x|<1,
$$

справедлива оценка

$$
v\left(\varepsilon^{q-1} \tau_{k}+0\right) \geqslant v\left(\varepsilon^{q-1} \tau_{k}\right)+\widehat{g}\left(v\left(\varepsilon^{q-1} \tau_{k}\right)\right)
$$

при всех $\tau_{k} \in\left[t_{0}, \infty\right)$, для которых решение задачи Коши $u\left(t ; t_{0}, \psi_{0}^{K} w\right)$ существует.

Из [21; лемма 1] следует неравенство

$$
0 \leqslant u\left(t ; t_{0}, \psi_{0}^{K} w\right) \leqslant \psi^{K}\left(t ; t_{0}, \psi_{0}\right) w,
$$

справедливое при всех $t \in\left[t_{0}, t^{*}\right)$, где $t^{*}-$ правый конец максимального интервала существования решения $u\left(t ; t_{0}, \psi_{0}^{K} w\right)$ задачи Коши для системы сравнения (5.2).

Определим норму в пространстве $\mathbb{R}^{s}$ согласно [25] по формуле

$$
\|x\|_{w}=\inf \{\beta \geqslant 0 \mid-\beta w \leqslant x \leqslant \beta w\} .
$$

Вследствие условия Важевского для функции $\widehat{f}(u)$ и предположений относительно функции $\widehat{g}(u)$ можно указать достаточно малое положительное число $\delta_{4}$, $\delta_{4}<\delta_{3}, \delta_{4}<\left\|\delta_{3}^{K}\right\|_{w}$, такое, что при всех $u_{0} \geqslant 0,\left\|u_{0}\right\|_{w}<\delta_{4}$ выполняется неравенство

$$
0 \leqslant u\left(t ; t_{0}, u_{0}\right) \leqslant u\left(t ; t_{0},\left\|u_{0}\right\|_{w} w\right) \leqslant u\left(t ; t_{0}, \delta_{3}^{K} w\right) \leqslant \psi^{K}\left(t ; t_{0}, \delta_{3}\right) w
$$


при всех тех $t \in\left[t_{0}, t^{* *}\right), t^{* *} \leqslant t^{*}$, для которых существует решение задачи Коши $u\left(t ; t_{0}, u_{0}\right)$. Из этого неравенства следует оценка

$$
\left\|u\left(t ; t_{0}, u_{0}\right)\right\|_{w} \leqslant\left\|\psi^{K}\left(t ; t_{0}, \delta_{3}\right)\right\|_{w}, \quad t \in\left[t_{0}, t^{* *}\right) .
$$

Если $t^{* *}<+\infty$, то в силу утверждения из [15; теорема 2.1] $\left\|u\left(t ; t_{0}, u_{0}\right)\right\|_{w} \rightarrow$ $+\infty$ при $t \rightarrow t^{* *}$, что противоречит неравенству (5.5). Это противоречие доказывает, что решение $u\left(t ; t_{0}, u_{0}\right)$ бесконечно продолжимо на интервал $\left[t_{0},+\infty\right)$. Таким образом, оценка (5.5) выполняется при всех $t \geqslant t_{0}$, откуда и следует выполнение необходимой априорной оценки.

СледствиЕ 1. Условия теоремы 2 гарантируют выполнение равномерной по $\varepsilon \in(0,1]$ априорной оценки решений вида (2.4) для однопараметрического семейства систем (2.3), а также асимптотическую устойчивость состояния равновесия $y=0$ систем (2.2).

5.2. Однородные системы, близкие к периодическим. Рассмотрим нелинейную однородную систему

$$
\begin{gathered}
\frac{d x}{d t}=f(x), \quad t \neq \tau_{k}, \\
\Delta x(t)=g(x(t)), \quad t=\tau_{k},
\end{gathered}
$$

где $x \in \mathbb{R}^{n}, f(x), g(x)$ - однородные функции нечетного измерения $\mu$. Установим условия, при которых для решений системы (5.6) выполняется априорная оценка $(2.4)$ с показателем $\sigma=1 /(\mu-1)$. Предположим, что существует положительное число $\theta$, для которого состояние равновесия $y=0$ системы обыкновенных дифференциальных уравнений

$$
\frac{d y}{d t}=\theta f(y)+g(y)
$$

где $y \in \mathbb{R}^{n}$, является асимптотически устойчивым. Тогда согласно результатам работ [7], [9] для достаточно большого натурального нечетного числа $m$ существует положительно определенная форма $W_{0}(x)$ порядка $m$ такая, что для некоторой положительно определенной формы $V_{0}(x)$ порядка $m-\mu+1$ справедливо уравнение

$$
\left(\nabla_{x} V_{0}(x)\right)^{T}(\theta f(x)+g(x))=-W_{0}(x) .
$$

Рассмотрим вспомогательную функцию вида

$$
V(t, x)=V_{0}(x)+V_{1}(t, x),
$$

где $V_{1}(t, x)$ форма по $x$ измерения $m$, коэффициенты которой суть ограниченные по $t$ функции, и

$$
V_{1}\left(\tau_{k}, x\right)=\lim _{t \rightarrow \tau_{k}-0} V_{1}(t, x), \quad V_{1}\left(\tau_{k}+0, x\right)=\lim _{t \rightarrow \tau_{k}+0} V_{1}(t, x) .
$$

Пусть $W(t, x)$ - некоторая однородная форма по $x$ измерения $m$, коэффициенты которой суть ограниченные по $t$ функции, и

$$
W\left(\tau_{k}, x\right)=\lim _{t \rightarrow \tau_{k}-0} W(t, x), \quad W\left(\tau_{k}+0, x\right)=\lim _{t \rightarrow \tau_{k}+0} W(t, x) .
$$


Функции $V(t, x)$ и $W(t, x)$ выберем из условий

$$
\begin{gathered}
\left(\nabla_{x} V_{0}(x)\right)^{T} f(x)+\frac{\partial V_{1}}{\partial t}=-W(t, x), \quad t \in\left(\tau_{k}, \tau_{k+1}\right] \\
\left(\nabla_{x} V_{0}(x)\right)^{T} g(x)+V_{1}\left(\tau_{k}+0, x\right)-V_{1}\left(\tau_{k}, x\right)=0
\end{gathered}
$$

Дополнительно введем условие $V_{1}\left(\tau_{k+1}+0, x\right)=V_{1}\left(\tau_{k}+0, x\right)=0, k \in \mathbb{N}$. Тогда уравнения (5.9) сводятся к одному уравнению

$$
\left(\nabla_{x} V_{0}(x)\right)^{T}\left(f(x)\left(\tau_{k+1}-\tau_{k}\right)+g(x)\right)=-\int_{\tau_{k}}^{\tau_{k+1}} W(t, x) d t .
$$

Таким образом, необходимо подобрать функции $V_{0}(x)$ и $W(t, x)$ так, чтобы удовлетворялось уравнение (5.10).

Пусть $\tau_{k+1}-\tau_{k}=\theta+\eta_{k}$. Функцию $W(t, x)$ будем искать в виде ряда по степеням $\eta_{k}$

$$
W(t, x)=W_{0}(x)+\eta_{k} W_{1}(x)+\cdots+\eta_{k}^{l} W_{l}(x)+\cdots, \quad t \in\left(\tau_{k}, \tau_{k+1}\right] .
$$

Подставляя это выражение в уравнение (5.10), получим последовательность уравнений

$$
\begin{aligned}
& W_{1}(x)=-\frac{1}{\theta}\left(W_{0}(x)+\left(\nabla_{x} V_{0}\right)^{T} f(x)\right), \\
& W_{l+1}(x)=-\frac{1}{\theta} W_{l}(x), \quad l=1,2, \ldots .
\end{aligned}
$$

Как следствие, из представления (5.11) получим

$$
W(t, x)=W_{0}(x)+W_{1}(x)\left(\eta_{k}-\frac{\eta_{k}^{2}}{\theta}+\cdots+(-1)^{l} \frac{\eta_{k}^{l+1}}{\theta^{l}}+\cdots\right), \quad t \in\left(\tau_{k}, \tau_{k+1}\right] .
$$

Условие сходимости ряда в правой части этого равенства состоит в выполнении неравенства $0<\tau_{k+1}-\tau_{k}<2 \theta$. В этом случае получим представление

$$
\begin{aligned}
W(t, x) & =W_{0}(x)+\frac{\eta_{k} \theta}{\eta_{k}+\theta} W_{1}(x) \\
& =\frac{\theta}{\tau_{k+1}-\tau_{k}} W_{0}(x)-\frac{\tau_{k+1}-\tau_{k}-\theta}{\tau_{k+1}-\tau_{k}}\left(\nabla_{x} V_{0}(x)\right)^{T} f(x), \quad t \in\left(\tau_{k}, \tau_{k+1}\right] .
\end{aligned}
$$

Предположим, что функция $W(t, x)$ является положительно определенной. Тогда существует положительная постоянная $\eta_{0}$ такая, что $W(t, x) \geqslant \eta_{0}\|x\|^{m}$ при всех $t \geqslant t_{0}$.

Из первого уравнения системы (5.9) находим

$$
V_{1}(t, x)=-\left(\left(\nabla_{x} V_{0}(x)\right)^{T} f(x)+\int_{\tau_{k}}^{\tau_{k+1}} W(s, x) d s\right)\left(t-\tau_{k}\right), \quad t \in\left(\tau_{k}, \tau_{k+1}\right] .
$$

Функция $V(t, x)$ является положительно определенной в некоторой достаточно малой окрестности точки $x=0$ и допускает бесконечно малый высший предел. 
Действительно, при $t \in\left[\tau_{k}, \tau_{k+1}\right]$ справедливы неравенства

$$
\begin{gathered}
\left|V_{1}(t, x)\right| \leqslant \theta_{2}\left(\left|\left(\nabla_{x} V_{0}(x)\right)^{T} f(x)\right|+\theta_{2} \sup _{s \in\left[\tau_{k}, \tau_{k+1}\right]}|W(s, x)|\right) \\
\sup _{s \in\left[\tau_{k}, \tau_{k+1}\right]}|W(s, x)| \leqslant \frac{\theta}{\tau_{k+1}-\tau_{k}}\left|W_{0}(x)\right|+\frac{\tau_{k+1}-\tau_{k}-\theta}{\tau_{k+1}-\tau_{k}}\left|\left(\nabla_{x} V_{0}(x)\right)^{T} f(x)\right| \\
\leqslant \frac{\theta}{\theta_{1}}\left|W_{0}(x)\right|+\frac{\theta_{2}-\theta}{\theta_{1}}\left|\left(\nabla_{x} V_{0}(x)\right)^{T} f(x)\right| .
\end{gathered}
$$

Поскольку функции $W_{0}(x)$ и $\left(\nabla_{x} V_{0}(x)\right)^{T} f(x)$ являются формами $m$-го измерения, то существует постоянная $M>0$ такая, что

$$
\left|W_{0}(x)\right| \leqslant M\|x\|^{m}, \quad\left|\left(\nabla_{x} V_{0}(x)\right)^{T} f(x)\right| \leqslant M\|x\|^{m} .
$$

Поэтому

$$
\left|V_{1}(t, x)\right| \leqslant M \theta_{2}\left(1+\frac{\theta_{2}^{2}}{\theta_{1}}\right)\|x\|^{m} .
$$

По предположению функция $V_{0}(x)$ является положительно определенной формой $(m-\mu+1)$-го измерения, поэтому существуют положительные постоянные $m_{i}, i=1,2$, такие, что

$$
m_{1}\|x\|^{m-\mu+1} \leqslant V_{0}(x) \leqslant m_{2}\|x\|^{m-\mu+1} .
$$

Поэтому

$$
V(t, x) \leqslant m_{2}\|x\|^{m-\mu+1}+M \theta_{2}\left(1+\frac{\theta_{2}^{2}}{\theta_{1}}\right)\|x\|^{m} .
$$

Отсюда следует, что функция $V(t, x)$ в достаточно малой окрестности точки $x=0$ допускает бесконечно малый высший предел. Из неравенства

$$
V(t, x) \geqslant m_{1}\|x\|^{m-\mu+1}\left(1-\frac{M \theta_{2}}{m_{1}}\left(1+\frac{\theta_{2}^{2}}{\theta_{1}}\right)\|x\|^{\mu-1}\right)
$$

следует, что в окрестности точки $x=0$

$$
\left\{x \in \mathbb{R}^{n} \mid\|x\|<\left(\frac{m_{1}}{2 M \theta_{2}\left(1+\theta_{2}^{2} / \theta_{1}\right)}\right)^{\frac{1}{\mu-1}}\right\}
$$

справедливо неравенство $V(t, x) \geqslant m_{1}\|x\|^{m-\mu+1} / 2$, что доказывает положительную определенность функции $V(t, x)$.

Таким образом, построенная функция $V(t, x)$ в достаточно малой окрестности $B_{R}$ состояния равновесия $x=0$ удовлетворяет условиям

$$
\begin{gathered}
\gamma_{1}\|x\|^{m-\mu+1} \leqslant V(t, x) \leqslant \gamma_{2}\|x\|^{m-\mu+1}, \\
\left.\frac{d V}{d t}(t, x)\right|_{(5.6)} \leqslant-\gamma_{3}\|x\|^{m}, \quad t \neq \tau_{k}, \\
V\left(\tau_{k}+0, x+g(x)\right)-V\left(\tau_{k}, x\right) \leqslant \gamma_{4}\|x\|^{m+\mu-1} .
\end{gathered}
$$

Нетрудно доказать, что из этих условий следует асимптотическая устойчивость состояния равновесия $x=0$ системы (5.6). Поэтому существует достаточно малое положительное число $\rho_{0}$ такое, что для всех решений $x(t)$ с начальными 
условиями $x\left(t_{0}\right)=x_{0} \in B_{\rho_{0}}$ выполняется включение $x(t) \in B_{R}$ при всех $t \geqslant t_{0}$. Из условий (5.12) и (5.13) следует, что

$$
\frac{d V(t, x(t))}{d t} \leqslant-\frac{\gamma_{3}}{\gamma_{2}^{m /(m-\mu+1)}} V^{\frac{m}{m-\mu+1}}(t, x(t)), \quad t \neq \tau_{k} .
$$

Обозначим

$$
\gamma_{3}^{\prime}=\frac{\gamma_{3}}{\gamma_{2}^{\frac{m}{m-\mu+1}}}, \quad \gamma_{4}^{\prime}=\frac{\gamma_{4}}{\gamma_{1}^{\frac{m+\mu-1}{m-\mu+1}}} .
$$

Тогда, интегрируя последнее неравенство, получим

$$
\begin{aligned}
& V(t, x(t)) \leqslant V\left(\tau_{k}+0, x\left(\tau_{k}+0\right)\right) \\
& \times\left[1+\frac{\gamma_{3}^{\prime}(\mu-1)}{m-\mu+1}\left(t-\tau_{k}\right) V^{\frac{\mu-1}{m-\mu+1}}\left(\tau_{k}+0, x\left(\tau_{k}+0\right)\right)\right]^{\frac{m-\mu+1}{1-\mu}}, \\
& t \in\left(\tau_{k}, \tau_{k+1}\right] .
\end{aligned}
$$

Из неравенства (5.14) находим, что

$$
\begin{aligned}
V\left(\tau_{k+1}+\right. & \left.0, x\left(\tau_{k+1}+0\right)\right) \leqslant V\left(\tau_{k+1}, x\left(\tau_{k+1}\right)\right)+\gamma_{4}^{\prime} V^{\frac{m+\mu-1}{m-\mu+1}}\left(\tau_{k+1}, x\left(\tau_{k+1}\right)\right) \\
\leqslant V & \left.\tau_{k}+0, x\left(\tau_{k}+0\right)\right) \\
\times & {\left[1+\frac{\gamma_{3}^{\prime}(\mu-1)}{m-\mu+1}\left(\tau_{k+1}-\tau_{k}\right) V^{\frac{\mu-1}{m-\mu+1}}\left(\tau_{k}+0, x\left(\tau_{k}+0\right)\right)\right]^{\frac{m-\mu+1}{1-\mu}} } \\
+ & \gamma_{4}^{\prime} V^{\frac{m+\mu-1}{m-\mu+1}}\left(\tau_{k}+0, x\left(\tau_{k}+0\right)\right) \\
\times & {\left[1+\frac{\gamma_{3}^{\prime}(\mu-1)}{m-\mu+1}\left(\tau_{k+1}-\tau_{k}\right) V^{\frac{\mu-1}{m-\mu+1}}\left(\tau_{k}+0, x\left(\tau_{k}+0\right)\right)\right]^{\frac{m+\mu-1}{1-\mu}} } \\
\leqslant V & \left.\tau_{k}+0, x\left(\tau_{k}+0\right)\right) \\
\times & {\left[1+\frac{\gamma_{3}^{\prime}(\mu-1)}{m-\mu+1} \theta_{1} V^{\frac{\mu-1}{m-\mu+1}}\left(\tau_{k}+0, x\left(\tau_{k}+0\right)\right)\right]^{\frac{m-\mu+1}{1-\mu}} } \\
+ & \gamma_{4}^{\prime} V^{\frac{m+\mu-1}{m-\mu+1}}\left(\tau_{k}+0, x\left(\tau_{k}+0\right)\right) \\
\times & {\left[1+\frac{\gamma_{3}^{\prime}(\mu-1)}{m-\mu+1} \theta_{1} V^{\frac{\mu-1}{m-\mu+1}}\left(\tau_{k}+0, x\left(\tau_{k}+0\right)\right)\right]^{\frac{m+\mu-1}{1-\mu}} . }
\end{aligned}
$$

Поэтому выполняется неравенство

$$
V\left(\tau_{k}+0, x\left(\tau_{k}+0\right)\right) \leqslant v_{k},
$$

где $v_{k}$ - решение разностного уравнения

$$
\begin{gathered}
v_{k+1}=v_{k}\left[1+c_{0} v_{k}^{\frac{\mu-1}{m-\mu+1}}\right]^{\frac{m-\mu+1}{1-\mu}}+\gamma_{4}^{\prime} v_{k}^{\frac{m+\mu-1}{m-\mu+1}}\left[1+c_{0} v_{k}^{\frac{\mu-1}{m-\mu+1}}\right]^{\frac{m+\mu-1}{1-\mu}}, \\
c_{0}=\frac{\gamma_{3}^{\prime}(\mu-1)}{m-\mu+1} \theta_{1} .
\end{gathered}
$$

Предполагая, что величина $v_{k}$ достаточно малая, разложим правую часть этого уравнения в ряд Тейлора

$$
v_{k+1}=v_{k}-\gamma_{3}^{\prime} \theta_{1} v_{k}^{\frac{m}{m-\mu+1}}+O\left(v_{k}^{\frac{m+\mu-1}{m-\mu+1}}\right) .
$$


Используя утверждение леммы 3, приходим к выводу о существовании постоянной $c_{1}$ и натурального числа $k_{0}$ таких, что при всех $k \geqslant k_{0}$ выполняется неравенство

$$
v_{k} \leqslant c_{1} k^{\frac{m-\mu+1}{1-\mu}} .
$$

Из этой оценки выводится неравенство

$$
\left\|x\left(\tau_{k}+0 ; t_{0}, x_{0}\right)\right\| \leqslant\left(\frac{c_{1}}{\gamma_{1}}\right)^{\frac{1}{m-\mu+1}} k^{\frac{1}{1-\mu}},
$$

из которого достаточно просто выводится необходимая априорная оценка (2.4) с показателем $\sigma=\frac{1}{\mu-1}$.

Итогом приведенных выше рассуждений является следующее утверждение.

Теорема 4. Предположим, что система дифберенииалъных уравнений (5.6) такова, что для заданной положительно определенной формы $W_{0}(x)$ существуют положительная постоянная $\theta$ и положительно определенная форма $V_{0}(x)$, удовлетворяющая системе дифберенииальных уравнений в частных производных (5.8), а последовательность моментов импульсного воздействия $\left\{\tau_{k}\right\}_{k=1}^{\infty}$ такова, что

$$
0<\theta_{1} \leqslant \tau_{k+1}-\tau_{k} \leqslant \theta_{2}, \quad \theta_{2}<2 \theta
$$

и форма

$$
W(t, x)=\frac{\theta}{\tau_{k+1}-\tau_{k}} W_{0}(x)-\frac{\tau_{k+1}-\tau_{k}-\theta}{\tau_{k+1}-\tau_{k}}\left(\nabla_{x} V_{0}(x)\right)^{T} f(x), \quad t \in\left(\tau_{k}, \tau_{k+1}\right],
$$

является положителъно определенной.

Тогда состояние равновесия $x=0$ однородной системы дифференииальных уравнений с импульсным воздействием (5.6) является асимптотически устойчивым и для ее решений выполняется априорная оценка (2.4) с показателем $\sigma=1 /(\mu-1)$.

Отметим, что условие положительной определенности формы $W(t, x)$ позволяет установить пределы малых отклонений величины $\tau_{k+1}-\tau_{k}$ от $\theta$, при которых устойчивость состояния равновесия $x=0$ системы (5.6) будет асимптотической, и при этом будет выполняться априорная оценка (2.4). В случае $n=2$ построение формы $V_{0}(x)$ по заданной форме $W_{0}(x)$ сводится к квадратурам (см. [7]).

\section{§6. Пример}

В качестве примера рассмотрим вопрос об устойчивости крупномасштабной импульсной системы

$$
\begin{gathered}
\frac{d \mathbf{x}_{s}}{d t}=\mathbf{f}_{s}\left(\mathbf{x}_{s}\right)+\sum_{j=1}^{m} \mathbf{R}_{s j}(t, \mathbf{x}), \quad t \neq \tau_{k}, \\
\Delta \mathbf{x}_{s}(t)=\mathbf{g}_{s}\left(\mathbf{x}_{s}(t)\right)+\sum_{j=1}^{m} \mathbf{Q}_{s j}^{(k)}(\mathbf{x}(t)), \quad t=\tau_{k},
\end{gathered}
$$


описывающей динамику системы, состоящей из $m$ взаимодействующих подсистем. Здесь $\mathbf{x}_{s} \in \mathbb{R}^{n_{s}}, \mathbf{x}=\left(\mathbf{x}_{1}^{T}, \ldots, \mathbf{x}_{m}^{T}\right)^{T} \in \mathbb{R}^{n}, n=\sum_{s=1}^{m} n_{s}, \mathbf{f}_{s}\left(\mathbf{x}_{s}\right)$ - непрерывно дифференцируемые однородные порядка $\mu_{s}, \mu_{s}>1$, функции, a $\mathbf{g}_{s}\left(\mathbf{x}_{s}\right)-$ непрерывные однородные порядка $\mu_{s}$ функции. Векторные функции $\mathbf{R}_{s j}(t, \mathbf{x})$ и $\mathbf{Q}_{s j}^{(k)}(\mathbf{x})$ - непрерывные при $t \geqslant t_{0}, k \in \mathbb{N},\|\mathbf{x}\|<H$ ( $H$ - положительная постоянная, $\|\cdot\|$ - евклидова норма), удовлетворяющие неравенствам

$$
\begin{gathered}
\left\|\mathbf{R}_{s j}(t, \mathbf{x})\right\| \leqslant c_{s j}\left\|\mathbf{x}_{1}\right\|^{\alpha_{s j}^{(1)}} \cdots\left\|\mathbf{x}_{m}\right\|^{\alpha_{s j}^{(m)}}, \quad c_{s j} \geqslant 0, \quad \alpha_{s j}^{(l)} \geqslant 0, \\
\left\|\mathbf{Q}_{s j}^{(k)}(\mathbf{x})\right\| \leqslant d_{s j}\left\|\mathbf{x}_{1}\right\|^{\beta_{s j}^{(1)}} \cdots\left\|\mathbf{x}_{m}\right\|^{\beta_{s j}^{(m)}}, \quad d_{s j} \geqslant 0, \quad \beta_{s j}^{(l)} \geqslant 0, \quad s, j, l=\overline{1, m} .
\end{gathered}
$$

Будем предполагать, что

$$
\mathbf{R}_{s j}(t, 0)=0, \quad \mathbf{Q}_{s j}^{(k)}(0)=0,
$$

т.е. система (6.1) имеет критическое состояние равновесия $\mathbf{x}=0$.

Относительно последовательности моментов импульсного воздействия $\left\{\tau_{k}\right\}_{k=1}^{\infty}$ предположим, что выполняется неравенство

$$
0<\theta_{1} \leqslant \tau_{k+1}-\tau_{k} \leqslant \theta_{2}<+\infty .
$$

Решение $\mathbf{x}\left(t ; t_{0}, \mathbf{x}_{0}\right)=\left(\mathbf{x}_{1}^{T}\left(t ; t_{0}, \mathbf{x}_{0}\right), \ldots, \mathbf{x}_{m}^{T}\left(t ; t_{0}, \mathbf{x}_{0}\right)\right)^{T}$ является непрерывно дифференцируемой функцией на множестве $\left[t_{0}, \omega^{+}\left(t_{0}, \mathbf{x}_{0}\right)\right) \backslash\left\{\tau_{k}\right\}_{k=1}^{\infty}$ и непрерывной слева при $t=\tau_{k}$, где $\omega^{+}\left(t_{0}, \mathbf{x}_{0}\right)$ - правый конец максимального интервала существования решения $\mathbf{x}\left(t ; t_{0}, \mathbf{x}_{0}\right)$.

Предположим, что нулевые решения $\mathbf{x}_{s}=0$ независимых подсистем

$$
\begin{aligned}
\frac{d \mathbf{x}_{s}}{d t} & =\mathbf{f}_{s}\left(\mathbf{x}_{s}\right), \quad t \neq \tau_{k}, \\
\Delta \mathbf{x}_{s}(t) & =\mathbf{g}_{s}\left(\mathbf{x}_{s}(t)\right), \quad t=\tau_{k},
\end{aligned}
$$

являются асимптотически устойчивыми и, более того, для решений соответствующих однопараметрических семейств однородных систем

$$
\begin{gathered}
\frac{d \mathbf{y}_{s}}{d \tau}=\mathbf{f}_{s}\left(\mathbf{y}_{s}\right), \quad \tau \neq \varepsilon^{\mu_{s}-1} \tau_{k}, \\
\Delta \mathbf{y}_{s}(\tau)=\varepsilon^{\mu_{s}-1} \mathbf{g}_{s}\left(\mathbf{y}_{s}(t)\right), \quad \tau=\varepsilon^{\mu_{s}-1} \tau_{k},
\end{gathered}
$$

выполняется априорная оценка решений. А именно существуют положительные постоянные $a_{s}, \sigma_{s}, \tau_{s}^{\prime}, r_{s}$, не зависящие от $\varepsilon \in(0,1]$, такие, что при всех $\tau \geqslant \tau_{0}+\tau_{m}^{\prime}, y_{0} \in \partial B_{r_{m}}$ выполняется неравенство

$$
\left\|\mathbf{y}_{s}\left(\tau ; \tau_{0}, \mathbf{y}_{s 0}, \varepsilon\right)\right\| \leqslant a_{s}\left(\tau-\tau_{0}\right)^{-\sigma_{s}} .
$$

Применение теоремы 1 приводит к следующим условиям асимптотической устойчивости состояния равновесия $\mathbf{x}_{s}=0$ крупномасштабной системы (6.1):

$$
\sum_{i=1}^{n} \frac{\alpha_{s j}^{(i)}}{\mu_{i}-1}>\frac{\mu_{s}}{\mu_{s}-1}, \quad \sum_{i=1}^{n} \frac{\beta_{s j}^{(i)}}{\mu_{i}-1}>\frac{\mu_{s}}{\mu_{s}-1} .
$$


В качестве конкретного примера рассмотрим двухкомпонентную систему с импульсным воздействием

$$
\begin{aligned}
& \frac{d x_{1}}{d t}=-a x_{1}^{3}+\varepsilon x_{1}^{2} x_{2}+\left(x_{1}^{2}+x_{2}^{2}\right)^{\frac{7}{6}} x_{3}^{4}, \\
& \frac{d x_{2}}{d t}=-a x_{2}^{3}+\varepsilon x_{1}^{3}+2\left(x_{1}^{2}+x_{2}^{2}\right)^{\frac{7}{6}} x_{3}^{4}, \\
& \frac{d x_{3}}{d t}=b x_{3}^{5}+\left(x_{1}^{2}+x_{2}^{2}\right)^{2} x_{3}^{\frac{4}{3}}, \quad t \neq \tau_{k}, \\
& \Delta x_{1}=c x_{1}^{3}+\varepsilon x_{1}^{2} x_{2}+2\left(x_{1}^{2}+x_{2}^{2}\right)^{\frac{7}{6}} x_{3}^{4}, \\
& \Delta x_{2}=c x_{2}^{3}+\varepsilon x_{1}^{3}+\left(x_{1}^{2}+x_{2}^{2}\right)^{\frac{7}{6}} x_{3}^{4}, \\
& \Delta x_{3}=-d x_{3}^{5}+\left(x_{1}^{2}+x_{2}^{2}\right)^{2} x_{3}^{\frac{4}{3}}, \quad t=\tau_{k} .
\end{aligned}
$$

Здесь $a, b, c, d-$ положительные параметры, $\varepsilon \in \mathbb{R}$. Примем

$$
\mathbf{x}_{1}=\left(x_{1}, x_{2}\right)^{T}, \quad \mathbf{x}_{2}=x_{3}
$$

Теорема 3 гарантирует выполнение априорных оценок для соответствующих семейств нелинейных систем и асимптотическую устойчивость состояний равновесия $\mathbf{x}_{s}=0, s=1,2$, независимых подсистем, если только выполняются неравенства

$$
-a \theta_{1}+|\varepsilon| \theta_{2}+c+|\varepsilon|<0, \quad b \theta_{2}-d<0 .
$$

Применение утверждения теоремы 1 позволяет заключить, что состояния равновесия $x_{1}=x_{2}=x_{3}=0$ системы (6.6) асимптотически устойчиво.

Отметим, что состояние равновесия $x_{1}=x_{2}=x_{3}=0$ соответствующей системы без импульсного воздействия

$$
\begin{aligned}
& \frac{d x_{1}}{d t}=-a x_{1}^{3}+\varepsilon x_{1}^{2} x_{2}+\left(x_{1}^{2}+x_{2}^{2}\right)^{\frac{7}{6}} x_{3}^{4}, \\
& \frac{d x_{2}}{d t}=-a x_{2}^{3}+\varepsilon x_{1}^{3}+2\left(x_{1}^{2}+x_{2}^{2}\right)^{\frac{7}{6}} x_{3}^{4}, \\
& \frac{d x_{3}}{d t}=b x_{3}^{5}+\left(x_{1}^{2}+x_{2}^{2}\right)^{2} x_{3}^{\frac{4}{3}}
\end{aligned}
$$

не является асимптотически устойчивым. Также и неподвижная точка $x_{1}=$ $x_{2}=x_{3}=0$ точечного отображения скачка

$$
\begin{aligned}
& \bar{x}_{1}=x_{1}+c x_{1}^{3}+\varepsilon x_{1}^{2} x_{2}+2\left(x_{1}^{2}+x_{2}^{2}\right)^{\frac{7}{6}} x_{3}^{4}, \\
& \bar{x}_{2}=x_{2}+c x_{2}^{3}+\varepsilon x_{1}^{3}+\left(x_{1}^{2}+x_{2}^{2}\right)^{\frac{7}{6}} x_{3}^{4}, \\
& \bar{x}_{3}=x_{3}-d x_{3}^{5}+\left(x_{1}^{2}+x_{2}^{2}\right)^{2} x_{3}^{\frac{4}{3}}
\end{aligned}
$$

не является устойчивой. Поэтому асимптотическая устойчивость состояния равновесия $x_{1}=x_{2}=x_{3}=0$ системы (6.6) является следствием сложного взаимодействия непрерывной и дискретной динамики. Этот же пример свидетельствует о достаточной общности полученных результатов. 


\section{$\S 7$. Обсуждение результатов}

Применение основной теоремы, полученной в настоящей работе, сопряжено с необходимостью предварительного доказательства априорной оценки (2.4), равномерной по параметру $\varepsilon \in(0,1]$, для решений однопараметрического семейства нелинейных импульсных систем (3.2). Такие оценки могут быть получены при использовании метода Ляпунова в сочетании с концепцией метода сравнения. В настоящей работе указан общий способ получения априорных оценок для семейства нелинейных квазиоднородных систем Важевского и для однородных систем, близких к периодическим. Устойчивость автономных систем обыкновенных дифференциальных уравнений со свойством Важевского были предметом исследования в работе [26], которые были развиты в ряде работ [27]-[30]. Вместе с этим результаты, полученные в этих работах, позволяют установить лишь сам факт устойчивости или неустойчивости состояния равновесия, не давая никакой информации об оценках переходных процессов. Наряду с этим в работе [13] предложен иной метод исследования импульсных систем Важевского, который позволил не только установить факт устойчивости, но и также указать общий метод получения необходимых априорных оценок решений.

Отметим также, что отсутствие свойства автомодельности решений квазиоднородной импульсной системы приводит к отсутствию глобальности свойства асимптотической устойчивости. Поэтому актуальной для дальнейшего исследования является проблема оценки области притяжения для квазиоднородных импульсных систем.

\section{Список литературы}

[1] А. М. Ляпунов, Общая задача об устойчивости движения, Гостехиздат, М.-Л., 1950, 471 с.; англ. пер.: A. M. Lyapunov, The general problem of the stability of motion, Taylor \& Francis, Ltd., London, 1992, x+270 pp.

[2] Л.Г. Хазин, Э.Э. Шноль, Устойчивость критических положений равновесия, Изд-во НЦБИ АН СССР, Пущино, 1985, 216 с.; англ. пер.: L. G. Khazin, È. Ė Shnol', Stability of critical equilibrium states, Nonlinear Sci. Theory Appl., Manchester Univ. Press, Manchester, 1991, xii+208 pp.

[3] А. Я. Савченко, А. О. Игнатьев, Некоторые задачи устойчивости неавтономных динамических систем, Наукова думка, К., 1989, 304 с.

[4] Г. В. Каменков, Избранные труды, т. I, II, Наука, М., 1971, 258 рр., 214 с.

[5] Ф. Хартман, Обыкновенные дифференииальные уравнения, Мир, М., 1970, 720 с.; пер. с англ.: Ph. Hartman, Ordinary differential equations, John Wiley \& Sons, Inc., New York-London-Sydney, 1964, xiv+612 pp.

[6] В.А. Плисс, "Принцип сведения в теории устойчивости движения", Изв. АН СССР. Серия матем., 28:6 (1964), 1297-1324.

[7] В. И. Зубов, Устойчивость движения (методъ Ляпунова и их применение), 2-е изд., Высшая школа, М., 1984, 232 с.

[8] И.Г. Малкин, Теория устойчивости движения, 2-е изд., Наука, М., 1966, 530 с.; нем. пер. 1-го изд.: J. G. Malkin,, Theorie der Stabilität einer Bewegung, R. Oldenbourg, München, 1959, xiii+402 pp.

[9] Н.Н. Красовский, Некоторые задачи теории устойчивости движения, Физматгиз, М., 1959, 211 с.; англ. пер.: N. N. Krasovskii, Stability of motion. Applications of 
Lyapunov's second method to differential systems and equations with delay, Stanford Univ. Press, Stanford, Calif., 1963, vi+188 pp.

[10] Н. Н. Красовский, "Об устойчивости по первому приближению”, ПМM, 19 (1955), $516-530$.

[11] В.И. Зубов, Математические методы исследования систем автоматического регулирования, Судпромгиз, Л., 1959, 324 с.; англ. пер.: V.I. Zubov, Mathematical methods for the study of automatic control systems, Pergamon Press, New York-Oxford-London-Paris; Jerusalem Academic Press, Jerusalem, 1962, vii+327 pp.

[12] О. С. Черникова, "Принцип сведения для систем дифференциальных уравнений с импульсным воздействием", Укр. матем. журн., 34:5 (1982), 601-607; англ. пер.: O.S. Chernikova, "The reduction principle for systems of differential equations with impulse action", Ukrainian Math. J., 34:5 (1982), 487-492.

[13] А.И. Двирный, В.И. Слынько, “Аналог критического случая устойчивости Г. В. Каменкова для нестационарных систем дифференциальных уравнений с импульсным воздействием", Укр. матем. вестник, 9:3 (2012), 318-340; англ. пер.: A.I. Dvirny, V.I. Slyn'ko, "An analog to Kamenkov's critical stability case for nonstationary systems of impulsive differential equations", J. Math. Sci., 188:2 (2013), 85-101.

[14] А.И. Двирный, В.И. Слынько, "Устойчивость решений дифференциальных уравнений с импульсным воздействием в критических случаях", Cиб. матем. журн., 52:1 (2011), 70-80; англ. пер.: A. I. Dvirny̌̃, V.I. Slyn'ko, "Stability of solutions to impulsive differential equations in critical cases", Siberian Math. J., 52:1 (2011), 54-62.

[15] А. М. Самойленко, Н. А. Перестюк, Дифференииальные уравнения с импульсным воздействием, Вища школа, Киев, 1987, 288 с.; англ. пер.: А. M. Samoilenko, N. A. Perestyuk, Impulsive differential equations, World Sci. Ser. Nonlinear Sci. Ser. A Monogr. Treatises, 14, World Scientific Publishing Co., Inc., River Edge, NJ, 1995, ix +462 pp.

[16] A. O. Ignatyev, "On the stability of invariant sets of systems with impulse effect", Nonlinear Anal., 69:1 (2008), 53-72.

[17] В. В. Козлов, С.Д. Фурта, Асимптотики решений сильно нелинейных систем дифференциалъных уравнений, 2-е изд., НИЦ "Регулярная и хаотическая динамика Ижевский Институт компьютерных исследований, М.-Ижевск, 2009, 312 с.; англ. пер.: V. V. Kozlov, S.D. Furta, Asymptotic solutions of strongly nonlinear systems of differential equations, Springer Monogr. Math., Springer, Heidelberg, 2013, $\mathrm{xx}+262 \mathrm{pp}$.

[18] А. О. Игнатьев, "Метод функций Ляпунова в задачах устойчивости решений систем дифференциальных уравнений с импульсным воздействием", Матем. сб., 194:10 (2003), 117-132; англ. пер.: A. O. Ignat'yev, "Method of Lyapunov functions in problems of stability of solutions", Sb. Math., 194:10 (2003), 1543-1558.

[19] Р. И. Гладилина, А. О. Игнатьев, "О необходимых и достаточных условиях асимптотической устойчивости импульсных систем", Укр. матем. журнал, 55:8 (2003), 1035-1043; англ. пер.: R. I. Gladilina, A. O. Ignat'ev, "On necessary and sufficient conditions for the asymptotic stability of impulsive systems", Ukrainian Math. J., 55:8 (2003), 1254-1264.

[20] В.В. Румянцев, А.С. Озиранер, Устойчивость и стабилизация движения по отношению к части переменных, Наука, М., 1987, 255 с.

[21] V. Lakshmikantham, D. D. Bainov, P. S. Simeonov, Theory of impulsive differential equations, Ser. Modern Appl. Math., 6, World Scientific Publishing Co., Inc., Teaneck, NJ, 1989, xii +273 c. 
[22] А. И. Двирный, В.И. Слынько, "Устойчивость решений монотонных дифференциальных уравнений с импульсным воздействием", Дифферени. уравнения, 48:3 (2012), 316-325; англ. пер.: A. I. Dvirnyi, V. I. Slyn'ko, "Stability of solutions of monotone systems of impulsive differential equations", Differ. Equ., 48:3, 318-327.

[23] А. И. Двирный, В. И. Слынько, "Об устойчивости решений квазиоднородных монотонных импульсных систем”, Тр. Ин-та прикл. математики и механики, 24 (2012), 99-115.

[24] Г. Полиа, Г. Сегё, Задачи и теоремь из анализа, 2-е изд., ГИТТЛ, М., 1956, 391 с., 431 с.; пер. с нем.: G. Pólya, G. Szegö, Aufgaben und Lehrsätze aus der Analysis, v. 1, 2, 2te Aufl., Grundlehren Math. Wiss., XIX, XX, Springer-Verlag, Berlin-Göttingen-Heidelberg, 1954, xvi+338 pp., x+407 pp.

[25] М. А. Красносельский, Е.А. Лифшиц, А. В. Соболев, Позитивные линейные системы: метод положительных операторов, Наука, М., 1985, 256 с.; англ. пер.: M. A. Krasnosel'skij, Je. A. Lifshits, A. V. Sobolev, Positive linear systems. The method of positive operators, Sigma Ser. Appl. Math., 5, Heldermann Verlag, Berlin, 1989, viii+354 pp.

[26] А.А. Мартынюк, А. Ю. Оболенский, Исследование устойчивости автономных систем сравнения, Препринт 78.28, Ин-т математики АН УССР, Киев, 1978.

[27] А.Ю. Оболенский, "Об устойчивости систем сравнения”, Доп. АН УРСР, 8 (1979), 607-611.

[28] А. Ю. Оболенский, "Об устойчивости линейных систем сравнения”, Mam. физика и нелинейн. механика, 1 (1984), 51-55.

[29] А. Ю. Оболенский, Критерии устойчивости движения некоторых нелинейных систем, Феникс, К., 2010, 228 с.

[30] А.Ю. Оболенский, "Об устойчивости решений автономных систем Важевского с запаздыванием", Укр. матем. журн., 35:5 (1983), 574-579; англ. пер.: A. Yu. Obolenskii, "The stability of solutions of autonomous Wazewski systems with delayed action", Ukrainian Math. J., 35:5 (1983), 486-492.

\section{Александр Иванович Двирный}

(Aleksandr I. Dvirny)

Hadmark University College, Norway

E-mail: dvirny@mail.ru

\section{Виталий Иванович Слынько}

(Vitalij I. Slyn'ko)

Институт механики им. С. П. Тимошенко

НАН Украины, г. Киев, Украина

E-mail: vitstab@ukr.net
Поступила в редакцию 24.03.2013 и 09.12.2013 